\title{
Convolution of Lorentz Invariant
}

\section{Ultradistributions and Field Theory *}

\author{
C.G.Bollini and M.C.Rocca \\ Departamento de Física, Fac. de Ciencias Exactas, \\ Universidad Nacional de La Plata. \\ C.C. 67 (1900) La Plata. Argentina.
}

September 1, 2003

\begin{abstract}
In this work, a general definition of convolution between two arbitrary four dimensional Lorentz invariant (fdLi) Tempered Ultradistributions is given, in both: Minkowskian and Euclidean Space (Spherically symmetric tempered ultradistributions).

* This work was partially supported by Consejo Nacional de Investigaciones Cientificas and Comisión de Investigaciones Científicas de la Pcia. de Buenos Aires; Argentina.
\end{abstract}


The product of two arbitrary fdLi distributions of exponential type is defined via the convolution of its corresponding Fourier Transforms.

Several examples of convolution of two fdLi Tempered Ultradistributions are given. In particular we calculate exactly the convolution of two Feynman's massless propagators.

An expression for the Fourier Transform of a Lorentz invariant Tempered Ultradistribution in terms of modified Bessel distributions is obtained in this work (Generalization of Bochner's formula to Minkowskian space).

At the same time, and in a previous step used for the deduction of the convolution formula, we obtain the generalization to the Minkowskian space, of the dimensional regularization of the perturbation theory of Green Functions in the Euclidean configuration space given in ref.[12]. As an example we evaluate the convolution of two n-dimensional complex-mass Wheeler's propagators.

PACS: 03.65.-w, 03.65.Bz, 03.65.Ca, 03.65.Db. 


\section{Introduction}

The question of the product of distributions with coincident point singularities is related in Field Theory, to the asymptotic behavior of loop integrals of propagators.

From a mathematical point of view, practically all definitions lead to limitations on the set of distributions that can be multiplied together to give another distribution of the same kind.

The properties of ultradistributions (ref. [1, 2]) are well adapted for their use in Field Theory. In this respect we have shown (ref. [3]) that it is possible to define in one dimensional space, the convolution of any pair of tempered ultradistributions, giving as a result another tempered ultradistribution. The next step is to consider the convolution of any pair of tempered ultradistribution in n-dimensional space. This follows from the formula obtained in ref.[3] for one dimensional space (See ref. [4].)

However, the resultant formula is rather complex to be used in practical applications and calculus. Then, for applications, it is convenient to consider the convolution of any two tempered ultradistributions which are even in the variables $k^{0}$ y $\rho$ (See ref. 4 .).

A further step is to consider the convolution of two Lorentz invariant 
tempered ultradistributions (See Section 7)

Ultradistributions also have the advantage of being representable by means of analytic functions. So that, in general, they are easier to work with them and, as we shall see, have interesting properties. One of those properties is that Schwartz tempered distributions are canonical and continuously injected into tempered ultradistributions and as a consequence the Rigged Hilbert Space with tempered distributions is canonical and continuously included in the Rigged Hilbert Space with tempered ultradistributions.

This paper is organized as follow: in sections 2 and 3 we define the Distributions of Exponential Type and the Fourier transformed Tempered Ultradistributions. Each of them is part of a Guelfand's Triplet ( or Rigged Hilbert Space [5] ) together with their respective duals and a "middle term" Hilbert space. In section 4 we give a general expression for the Fourier transform of a spherically symmetric tempered ultradistributions and some examples of it. In section 5 we obtain the expression for the Fourier transform of Lorentz invariant tempered ultradistributions and we give some examples of its use. In section 6 , we give the general formula for the convolution of two spherically symmetric tempered ultradistributions and followed by some examples. In particular we evaluate exactly the convolution of two Feynman's massless 
propagators. In section 7 we treat the convolution of two Lorentz invariant tempered ultradistributions in Minkowskian space. In subsection 1, we give the generalization to Minkowskian space of the "dimensional regularization in configuration space" obtained in ref.[13. As an example of its use we evaluate convolution of two complex mass Wheeler's propagators. In subsection 2 we treat the central topic of this paper: the formula for the convolution of two Lorentz invariant tempered ultradistributions. Finally, section 8 is reserved for a discussion of the principal results.

\section{Distributions of Exponential Type}

For the sake of the reader we shall present a brief description of the principal properties of Tempered Ultradistributions.

Notations. The notations are almost textually taken from ref [2]. Let $\mathbb{R}^{\mathfrak{n}}\left(\right.$ res. $\left.\mathbb{C}^{\mathfrak{n}}\right)$ be the real (resp. complex) n-dimensional space whose points are denoted by $x=\left(x_{1}, x_{2}, \ldots, x_{n}\right)\left(\operatorname{resp} z=\left(z_{1}, z_{2}, \ldots, z_{n}\right)\right)$. We shall use the notations:

(i) $x+y=\left(x_{1}+y_{1}, x_{2}+y_{2}, \ldots, x_{n}+y_{n}\right) ; \alpha x=\left(\alpha x_{1}, \alpha x_{2}, \ldots, \alpha x_{n}\right)$

(ii) $x \geqq 0$ means $x_{1} \geqq 0, x_{2} \geqq 0, \ldots, x_{n} \geqq 0$ 


$$
\begin{aligned}
& \text { (iii) } x \cdot y=\sum_{j=1}^{n} x_{j} y_{j} \\
& (i V)|x|=\sum_{j=1}^{n}\left|x_{j}\right|
\end{aligned}
$$

Let $\mathbb{N}^{\mathfrak{n}}$ be the set of $\mathrm{n}$-tuples of natural numbers. If $p \in \mathbb{N}^{\mathfrak{n}}$, then $p=\left(p_{1}, p_{2}, \ldots, p_{n}\right)$, and $p_{j}$ is a natural number, $1 \leqq j \leqq n . p+q$ denote $\left(p_{1}+q_{1}, p_{2}+q_{2}, \ldots, p_{n}+q_{n}\right)$ and $p \geqq q$ means $p_{1} \geqq q_{1}, p_{2} \geqq q_{2}, \ldots, p_{n} \geqq q_{n}$. $x^{p}$ means $x_{1}^{p_{1}} x_{2}^{p_{2}} \ldots x_{n}^{p_{n}}$. We shall denote by $|p|=\sum_{j=1}^{n} p_{j}$ and by $D^{p}$ we denote the differential operator $\partial^{p_{1}+p_{2}+\ldots+p_{n}} / \partial x_{1}{ }^{p_{1}} \partial x_{2}{ }^{p_{2}} \ldots \partial x_{n}{ }^{p_{n}}$

For any natural $k$ we define $x^{k}=x_{1}^{k} x_{2}^{k} \ldots x_{n}^{k}$ and $\partial^{k} / \partial x^{k}=\partial^{n k} / \partial x_{1}^{k} \partial x_{2}^{k} \ldots \partial x_{n}^{k}$ The space $\mathcal{H}$ of test functions such that $e^{p|x|}\left|D^{q} \phi(x)\right|$ is bounded for any $\mathrm{p}$ and $\mathrm{q}$ is defined ( ref. [2] ) by means of the countably set of norms:

$$
\|\hat{\phi}\|_{p}=\sup _{0 \leq q \leq p, x} e^{p|x|}\left|D^{q} \hat{\phi}(x)\right| \quad, \quad p=0,1,2, \ldots
$$

According to reference[6] $\mathcal{H}$ is a $\mathcal{K}\left\{\boldsymbol{M}_{\mathbf{p}}\right\}$ space with:

$$
M_{p}(x)=e^{(p-1)|x|}, \quad p=1,2, \ldots
$$

$\mathcal{K}\left\{\mathbf{e}^{(\mathbf{p}-\mathbf{1})|\boldsymbol{x}|}\right\}$ satisfies condition $(\mathcal{N})$ of Guelfand ( ref. [5] ). It is a countable Hilbert and nuclear space:

$$
\mathcal{K}\left\{\mathbf{e}^{(\mathbf{p}-1)|x|}\right\}=\mathcal{H}=\bigcap_{p=1}^{\infty} \mathcal{H}_{\mathrm{p}}
$$

where $\mathcal{H}_{\mathrm{p}}$ is obtained by completing $\mathcal{H}$ with the norm induced by the scalar 
product:

$$
<\hat{\phi}, \hat{\psi}>_{p}=\int_{-\infty}^{\infty} e^{2(p-1)|x|} \sum_{q=0}^{p} D^{q} \widehat{\phi}(x) D^{q} \hat{\psi}(x) d x ; \quad p=1,2, \ldots
$$

where $d x=d x_{1} d x_{2} \ldots d x_{n}$

If we take the usual scalar product:

$$
<\hat{\phi}, \hat{\psi}>=\int_{-\infty}^{\infty} \widehat{\phi}(x) \hat{\psi}(x) d x
$$

then $\mathcal{H}$, completed with (2.5), is the Hilbert space $\mathbf{H}$ of square integrable functions.

The space of continuous linear functionals defined on $\mathcal{H}$ is the space $\boldsymbol{\Lambda}_{\infty}$ of the distributions of the exponential type ( ref.[2] ).

The "nested space"

$$
\sqrt[I]{\mathbf{I}}=\left(\mathcal{H}, \mathrm{H}, \boldsymbol{\Lambda}_{\infty}\right)
$$

is a Guelfand's triplet ( or a Rigged Hilbert space [5] ).

In addition we have: $\mathcal{H} \subset \mathcal{S} \subset \mathbf{H} \subset \mathcal{S}^{\prime} \subset \boldsymbol{\Lambda}_{\infty}$, where $\mathcal{S}$ is the Schwartz space of rapidly decreasing test functions ( $\operatorname{ref}[7])$.

Any Guelfand's triplet $\boldsymbol{G}=\left(\boldsymbol{\Phi}, \mathbf{H}, \boldsymbol{\Phi}^{\prime}\right)$ has the fundamental property that a linear and symmetric operator on $\boldsymbol{\Phi}$, admitting an extension to a self-adjoint operator in $\mathbf{H}$, has a complete set of generalized eigen-functions in $\boldsymbol{\Phi}^{\prime}$ with real eigenvalues. 


\section{Tempered Ultradistributions}

The Fourier transform of a function $\hat{\phi} \in \mathcal{H}$ is

$$
\phi(z)=\frac{1}{2 \pi} \int_{-\infty}^{\infty} \bar{\phi}(x) e^{i z \cdot x} d x
$$

$\phi(z)$ is entire analytic and rapidly decreasing on straight lines parallel to the real axis. We shall call $\mathfrak{H}$ the set of all such functions.

$$
\mathfrak{H}=\mathcal{F}\{\mathcal{H}\}
$$

It is a $\mathcal{Z}\left\{\mathbf{M}_{\mathbf{p}}\right\}$ space ( ref. [6] ), countably normed and complete, with:

$$
M_{p}(z)=(1+|z|)^{p}
$$

$\mathfrak{H}$ is also a nuclear space with norms:

$$
\|\phi\|_{p \mathfrak{n}}=\sup _{z \in V_{\mathfrak{n}}}(1+|z|)^{p}|\phi(z)|
$$

where $V_{k}=\left\{z=\left(z_{1}, z_{2}, \ldots, z_{n}\right) \in \mathbb{C}^{\mathfrak{n}}:\left|\operatorname{Im} z_{j}\right| \leqq k, 1 \leqq j \leqq n\right\}$

We can define the usual scalar product:

$$
<\phi(z), \psi(z)>=\int_{-\infty}^{\infty} \phi(z) \psi_{1}(z) \mathrm{d} z=\int_{-\infty}^{\infty} \overline{\hat{\phi}}(x) \hat{\psi}(x) \mathrm{d} x
$$

where:

$$
\psi_{1}(z)=\int_{-\infty}^{\infty} \hat{\psi}(x) e^{-i z \cdot x} d x
$$


and $d z=d z_{1} d z_{2} \ldots d z_{n}$

By completing $\mathfrak{H}$ with the norm induced by (3.5) we get the Hilbert space of square integrable functions.

The dual of $\mathfrak{H}$ is the space $\mathcal{U}$ of tempered ultradistributions ( ref. [2] ). In other words, a tempered ultradistribution is a continuous linear functional defined on the space $\mathfrak{H}$ of entire functions rapidly decreasing on straight lines parallel to the real axis.

The set $\mathfrak{d}=(\mathfrak{H}, \mathbf{H}, \mathcal{U})$ is also a Guelfand's triplet.

Moreover, we have: $\mathfrak{H} \subset \mathcal{S} \subset \mathbf{H} \subset \mathcal{S}^{\prime} \subset \mathcal{U}$.

$\mathcal{U}$ can also be characterized in the following way ( ref.[2] ): let $\mathcal{A}_{\boldsymbol{\omega}}$ be the space of all functions $F(z)$ such that:

I- $\mathrm{F}(z)$ is analytic for $\left\{z \in \mathbb{C}^{\mathfrak{n}}:\left|\operatorname{Im}\left(z_{1}\right)\right|>p,\left|\operatorname{Im}\left(z_{2}\right)\right|>p, \ldots,\left|\operatorname{Im}\left(z_{n}\right)\right|>\right.$ p\}.

II- $\mathrm{F}(z) / z^{p}$ is bounded continuous in $\left\{z \in \mathbb{C}^{\mathfrak{n}}:\left|\operatorname{Im}\left(z_{1}\right)\right| \geqq p,\left|\operatorname{Im}\left(z_{2}\right)\right| \geqq\right.$ $\left.p, \ldots,\left|\operatorname{Im}\left(z_{n}\right)\right| \geqq p\right\}$, where $p=0,1,2, \ldots$ depends on $F(z)$.

Let $\Pi$ be the set of all $z$-dependent pseudo-polynomials, $z \in \mathbb{C}^{\mathfrak{n}}$. Then $\mathcal{U}$ is the quotient space:

\section{III- $\mathcal{U}=\mathcal{A}_{\omega} / \Pi$}

By a pseudo-polynomial we understand a function of $z$ of the form 
$\sum_{s} z_{j}^{s} \mathrm{G}\left(z_{1}, \ldots, z_{j-1}, z_{j+1}, \ldots, z_{n}\right)$ with $\mathrm{G}\left(z_{1}, \ldots, z_{j-1}, z_{j+1}, \ldots, z_{n}\right) \in \mathcal{A}_{\boldsymbol{\omega}}$

Due to these properties it is possible to represent any ultradistribution as ( ref.[2]):

$$
\mathrm{F}(\phi)=<\mathrm{F}(z), \phi(z)>=\oint_{\Gamma} \mathrm{F}(z) \phi(z) \mathrm{d} z
$$

$\Gamma=\Gamma_{1} \cup \Gamma_{2} \cup \ldots \Gamma_{\mathrm{n}}$ where the path $\Gamma_{\mathrm{j}}$ runs parallel to the real axis from $-\infty$ to $\infty$ for $\operatorname{Im}\left(z_{j}\right)>\zeta, \zeta>p$ and back from $\infty$ to $-\infty$ for $\operatorname{Im}\left(z_{j}\right)<-\zeta$, $-\zeta<-$ p. ( $\Gamma$ surrounds all the singularities of $F(z)$ ).

Formula (3.6) will be our fundamental representation for a tempered ultradistribution. Sometimes use will be made of "Dirac formula" for ultradistributions ( ref.[1] ):

$$
F(z)=\frac{1}{(2 \pi i)^{n}} \int_{-\infty}^{\infty} \frac{f(t)}{\left(t_{1}-z_{1}\right)\left(t_{2}-z_{2}\right) \ldots\left(t_{n}-z_{n}\right)} d t
$$

where the "density" $f(t)$ is such that

$$
\oint_{\Gamma} F(z) \phi(z) d z=\int_{-\infty}^{\infty} f(t) \phi(t) d t
$$

While $F(z)$ is analytic on $\Gamma$, the density $f(t)$ is in general singular, so that the r.h.s. of (3.8) should be interpreted in the sense of distribution theory.

Another important property of the analytic representation is the fact that on $\Gamma, F(z)$ is bounded by a power of $z$ ( ref.[2] ):

$$
|\mathrm{F}(z)| \leq \mathrm{C}|z|^{\mathrm{p}}
$$


where $\mathrm{C}$ and $\mathrm{p}$ depend on $\mathrm{F}$.

The representation (3.6) implies that the addition of a pseudo-polynomial $\mathrm{P}(z)$ to $\mathrm{F}(z)$ do not alter the ultradistribution:

$$
\oint_{\Gamma}\{\mathrm{F}(z)+\mathrm{P}(z)\} \phi(z) \mathrm{d} z=\oint_{\Gamma} \mathrm{F}(z) \phi(z) \mathrm{d} z+\oint_{\Gamma} \mathrm{P}(z) \phi(z) \mathrm{d} z
$$

But:

$$
\oint_{\Gamma} \mathrm{P}(z) \phi(z) \mathrm{d} z=0
$$

as $\mathrm{P}(z) \phi(z)$ is entire analytic in some of the variables $z_{j}$ ( and rapidly decreasing ),

$$
\therefore \oint_{\Gamma}\{\mathrm{F}(z)+\mathrm{P}(z)\} \phi(z) \mathrm{d} z=\oint_{\Gamma} \mathrm{F}(z) \phi(z) \mathrm{d} z
$$

\section{The Fourier Transform in Euclidean Space}

The Fourier transform of a spherically symmetric function $\hat{f} \in \mathbf{H}$ is given, according to Bochner's formula by:

$$
f(k)=\frac{(2 \pi)^{\frac{v}{2}}}{k^{\frac{v-2}{2}}} \int_{0}^{\infty} \hat{f}(r) r^{\frac{v}{2}} \mathcal{J}_{\frac{v-2}{2}}(k r) d r
$$

where $r=x_{0}^{2}+x_{1}^{2}+\cdots+x_{v-1}^{2} ; k=k_{0}^{2}+k_{1}^{2}+\cdots+k_{v-1}^{2}$ and $\mathcal{J}_{\frac{v}{2}}$ is the Bessel function of order $v-2 / 2$. By the use of the equality

$$
\pi \mathcal{J}_{\frac{v-2}{2}}(z)=e^{-i \frac{\pi}{4} v} \mathcal{K}_{\frac{v-2}{2}}(-i z)+e^{i \frac{\pi}{4} v} \mathcal{K}_{\frac{v-2}{2}}(i z)
$$


where $\mathcal{K}$ is the modified Bessel function, (4.1) takes the form:

$$
\begin{gathered}
f(k)=2 \frac{(2 \pi)^{\frac{v-2}{2}}}{k^{\frac{v-2}{2}}} \int_{0}^{\infty} \hat{f}(r) r^{\frac{v}{2}}\left[e^{-i \frac{\pi}{4} v} \mathcal{K}_{\frac{v-2}{2}}(-i k r)+\right. \\
\left.e^{i \frac{\pi}{4} v} \mathcal{K}_{\frac{v-2}{2}}(i k r)\right] d r
\end{gathered}
$$

By performing the change of variables $x=r^{\frac{1}{2}}, \rho=k^{\frac{1}{2}}$ (4.1), and (4.3) can be re-written as:

$$
\begin{gathered}
f(\rho)=\pi \frac{(2 \pi)^{\frac{v-2}{2}}}{\rho^{\frac{v-2}{4}}} \int_{0}^{\infty} \hat{f}(x) x^{\frac{v-2}{4}} \mathcal{J}_{\frac{v-2}{2}}\left(\rho^{1 / 2} \chi^{1 / 2}\right) d x \\
f(\rho)=\frac{(2 \pi)^{\frac{v-2}{2}}}{\rho^{\frac{v-2}{4}}} \int_{0}^{\infty} \hat{f}(x) x^{\frac{v-2}{4}}\left[e^{-i \frac{\pi}{4} v} \mathcal{K}_{\frac{v-2}{2}}\left(-i x^{1 / 2} \rho^{1 / 2}\right)+\right. \\
\left.e^{i \frac{\pi}{4} v} \mathcal{K}_{\frac{v-2}{2}}\left(i x^{1 / 2} \rho^{1 / 2}\right)\right] d x
\end{gathered}
$$

Here we have taken $\rho=\gamma+i \sigma$ and

$$
\rho^{1 / 2}=\sqrt{\frac{\gamma+\sqrt{\gamma^{2}+\sigma^{2}}}{2}}+i \operatorname{Sgn}(\sigma) \sqrt{\frac{-\gamma+\sqrt{\gamma^{2}+\sigma^{2}}}{2}}
$$

We can extend (4.4) to the complex plane and obtain the corresponding ultradistribution. As a first step we calculate the Fourier antitrasfom of $\rho^{\frac{2-v}{4}} \mathcal{J}_{\frac{v-2}{2}}\left(\chi^{1 / 2} \rho^{1 / 2}\right)$. We have:

$$
\frac{1}{2 \pi} \int_{0}^{\infty} \rho^{\frac{2-v}{4}} \mathcal{J}_{\frac{v-2}{2}}\left(x^{1 / 2} \rho^{1 / 2}\right) e^{-i \rho t} d \rho=
$$




$$
\frac{e^{\frac{i \pi(v-4)}{8}}(t-i 0)^{\frac{v-4}{4}}}{\pi x^{1 / 2} \Gamma\left(\frac{v}{2}\right)} e^{\frac{i x}{8 t}} \mathcal{M}_{\frac{4-v}{4}, \frac{v-2}{4}}\left(-\frac{i x}{4 t}\right)
$$

We have used 6.631.(1) of ref. [9] ( $\mathcal{M}$ is the Whittaker function). Now we can use 9.233, (1), (2) of ref. [9] and write

$$
\begin{gathered}
\mathcal{M}_{\frac{4-v}{4}, \frac{v-2}{4}}\left(-\frac{i x}{4 t}\right)=\frac{\Gamma\left(\frac{v}{2}\right)}{\Gamma\left(\frac{v-2}{2}\right)} e^{\frac{i \pi(4-v)}{4}} \mathcal{W}_{\frac{v-4}{4}, \frac{v-2}{4}}\left(\frac{i x}{4 t}\right)+ \\
\Gamma\left(\frac{v}{2}\right) e^{\frac{i \pi(2-v)}{2}} \mathcal{W}_{\frac{4-v}{4}, \frac{v-2}{4}}\left(-\frac{i x}{4 t}\right) \quad t>0 . \\
\mathcal{M}_{\frac{4-v}{4}, \frac{v-2}{4}}\left(-\frac{i x}{4 t}\right)=\frac{\Gamma\left(\frac{v}{2}\right)}{\Gamma\left(\frac{v-2}{2}\right)} e^{\frac{i \pi(v-4)}{4}} \mathcal{W}_{\frac{v-4}{4}, \frac{v-2}{4}}\left(\frac{i x}{4 t}\right)+ \\
\Gamma\left(\frac{v}{2}\right) e^{\frac{i \pi(v-2)}{2}} \mathcal{W}_{\frac{4-v}{4}, \frac{v-2}{4}}\left(-\frac{i x}{4 t}\right) \quad t<0 .
\end{gathered}
$$

As a second step we calculate the complex Fourier transform of the second term of (4.7) using (4.8). We obtain:

$$
\begin{aligned}
& \mathcal{F}_{c}\left[\frac{e^{\frac{i \pi(v-4)}{8}}(t-i 0)^{\frac{v-4}{4}}}{\pi \chi^{1 / 2} \Gamma\left(\frac{v}{2}\right)} e^{\frac{i x}{8 t}} \mathcal{M}_{\frac{4-v}{4}, \frac{v-2}{4}}\left(-\frac{i x}{4 t}\right)\right](\rho)= \\
& \rho^{\frac{2-\gamma}{4}}\left\{\Theta[\Im(\rho)] e^{-\frac{i \pi v}{4}} \mathcal{K}_{\frac{v-2}{2}}\left(-i x^{1 / 2} \rho^{1 / 2}\right)-\Theta[-\mathfrak{I}(\rho)] e^{\frac{i \pi v}{4}} \mathcal{K}_{\frac{\nu-2}{2}}\left(i x^{1 / 2} \rho^{1 / 2}\right)+\right. \\
& \left.\frac{2^{\frac{4-v}{2} i}}{\Gamma\left(\frac{v-2}{2}\right)} \mathcal{S}_{\frac{v-4}{2}, \frac{v-2}{2}}\left(x^{1 / 2} \rho^{1 / 2}\right)\right\}
\end{aligned}
$$


where we have used 7.629,(1),(2) of ref. 9 and $\mathcal{S}$ is the Lommel function (ref.10, pag 349, formula 3). The corresponding ultradistribution is then defined as:

$$
\begin{gathered}
F(\rho)=\frac{(2 \pi)^{\frac{v-2}{2}}}{\rho^{\frac{v-2}{4}}} \int_{0}^{\infty} \hat{f}(x) x^{\frac{v-2}{4}}\left\{\Theta[\Im(\rho)] e^{-\frac{i \pi v}{4}} \mathcal{K}_{\frac{v-2}{2}}\left(-i x^{1 / 2} \rho^{1 / 2}\right)-\right. \\
\left.\Theta[-\Im(\rho)] e^{\frac{i \pi v}{4}} \mathcal{K}_{\frac{v-2}{2}}\left(i x^{1 / 2} \rho^{1 / 2}\right)\right\} d x+ \\
\frac{2 \pi^{\frac{v-2}{2}}}{\Gamma\left(\frac{v-2}{2}\right) \rho^{\frac{v-2}{4}}} \int_{0}^{\infty} \hat{f}(\chi) \chi^{\frac{v-2}{4}} \mathcal{S}_{\frac{v-4}{2}, \frac{v-2}{2}}\left(x^{1 / 2} \rho^{1 / 2}\right) d x
\end{gathered}
$$

When $v=2 n, n$ an entire number, $\rho^{\frac{2-v}{4}} \mathcal{S}_{\frac{v-4}{2}}, \frac{v-2}{2}$ is equivalent to zero. In fact

$$
\rho^{\frac{2-v}{4}} \mathcal{S}_{\frac{v-4}{2}, \frac{v-2}{2}}=\sum_{m=0}^{\frac{v-4}{2}} \frac{\left(\frac{v}{2}-m\right) !}{m !} 4^{\frac{v-2-4 m}{4}} x^{\frac{4 m+2-v}{4}} \rho^{\frac{2 m+2-v}{2}}
$$

(4.11) is a polynomial in $\rho^{-1}$. However when the volume element is taken into account that expression is transformed into a polynomial in $\rho$ which according to (3.10) is a null ultradistribution. Thus in this case the second integral in (4.10) vanishes and it becomes in:

$$
\begin{gathered}
F(\rho)=\frac{(2 \pi)^{\frac{v-2}{2}}}{\rho^{\frac{v-2}{4}}} \int_{0}^{\infty} \hat{f}(x) x^{\frac{v-2}{4}}\left[\Theta[\Im(\rho)] e^{-i \frac{\pi}{4} v} \mathcal{K}_{\frac{v-2}{2}}\left(-i x^{1 / 2} \rho^{1 / 2}\right)\right. \\
\left.-\Theta[-\mathfrak{I}(\rho)] e^{i \frac{\pi}{4} v} \mathcal{K}_{\frac{v-2}{2}}\left(i x^{1 / 2} \rho^{1 / 2}\right)\right] d x
\end{gathered}
$$


Note that the complex Fourier transform (4.12) is not merely the Fourier transform (4.5) in which the variable $\rho$ is considered to be a complex number. (4.12) gives the ultradistribution associated to $f(\rho)$. In the next section we shall see that formulae (4.5), (4.12) can be generalized to Minkowskian space.

When $\hat{f}$ is a spherically symmetric distribution of exponential type, we can use (4.10) to define its Fourier transform. In addition we can follow the treatment of ref. 11] to define the Fourier transform. Thus we have

$$
\int_{0}^{\infty} f(\rho) \phi(\rho) \rho^{\frac{v-2}{2}} d \rho=(2 \pi)^{v} \int_{0}^{\infty} \hat{f}(x) \hat{\phi}(x) x^{\frac{v-2}{2}} d x
$$

The corresponding tempered ultradistribution in the one-dimensional complex variable $\rho$ is obtained in the following way: let $\hat{g}(t)$ be defined as:

$$
\hat{g}(t)=\frac{1}{(2 \pi)^{v}} \int_{0}^{\infty} f(\rho) e^{-i \rho t} d \rho
$$

Then:

$$
F(\rho)=\Theta[\Im(\rho)] \int_{0}^{\infty} \hat{g}(t) e^{i \rho t} d t-\Theta[-\mathfrak{I}(\rho)] \int_{-\infty}^{0} \hat{g}(t) e^{i \rho t} d t
$$

or if we use Dirac's formula

$$
F(\rho)=\frac{1}{2 \pi i} \int_{0}^{\infty} \frac{f(t)}{t-\rho} d t
$$

The inversion formula $(v=2 n)$ for $F(\rho)$ is given by

$$
\hat{f}(x)=\frac{\pi}{(2 \pi)^{\frac{v+2}{2}} \chi^{\frac{v-2}{4}}} \oint_{\Gamma} F(\rho) \rho^{\frac{v-2}{4}} \mathcal{J}_{\frac{v-2}{2}}\left(\chi^{1 / 2} \rho^{1 / 2}\right) d \rho
$$


Note that the factor multiplying $F(\rho)$ is an entire function of $\rho$ for $v=2 n$. In this case the first term of (4.13) takes the form:

$$
\oint_{\Gamma} F(\rho) \phi(\rho) \rho^{\frac{v-2}{2}} d \rho=(2 \pi)^{v} \int_{0}^{\infty} \hat{f}(x) \hat{\phi}(x) x^{\frac{v-2}{2}} d x
$$

We can now define a spherically symmetric tempered ultradistribution as the complex Fourier transform of a spherically symmetric distribution of exponential type. Note that a spherically symmetric ultradistribution is not necessarily spherically symmetric in an explicit way.

We give now same examples of the use of Fourier transform.

\section{Examples}

As a first example we calculate the complex Fourier transform of $e^{\text {ar }}$ (where a is a complex number) for $v=2 n$. From (4.12) we write:

$$
\begin{gathered}
F(\rho)=\frac{(2 \pi)^{\frac{v-2}{2}}}{\rho^{\frac{v-2}{4}}} \int_{0}^{\infty} e^{a x^{1 / 2}} x^{\frac{v-2}{4}}\left\{\Theta[\Im(\rho)] e^{-\frac{i \pi v}{4}} \mathcal{K}_{\frac{v-2}{2}}\left(-i x^{1 / 2} \rho^{1 / 2}\right)-\right. \\
\left.\Theta[-\Im(\rho)] e^{\frac{i \pi v}{4}} \mathcal{K}_{\frac{v-2}{2}}\left(i x^{1 / 2} \rho^{1 / 2}\right) d x\right\}
\end{gathered}
$$

Now:

$$
\int_{0}^{\infty} e^{a x^{1 / 2}} x^{\frac{v-2}{4}} \mathcal{K}_{\frac{v-2}{2}}\left(-i x^{1 / 2} \rho^{1 / 2}\right)=2 \sqrt{\pi} e^{\frac{i \pi(v+2)}{4}} \frac{\Gamma(v)}{\Gamma\left(\frac{v+3}{2}\right)} \frac{\rho^{\frac{v-2}{4}}}{\left(\rho^{1 / 2}-i a\right)} \times
$$




$$
\begin{aligned}
& F\left(v, \frac{v-1}{2}, \frac{v+3}{2}, \frac{a-i \rho^{1 / 2}}{a+i \rho^{1 / 2}}\right) \quad \Im(\rho)>0 \\
& \int_{0}^{\infty} e^{a x^{1 / 2}} x^{\frac{v-2}{4}} \mathcal{K}_{\frac{v-2}{2}}\left(i x^{1 / 2} \rho^{1 / 2}\right)=2 \sqrt{\pi} e^{-\frac{i \pi(v+2)}{4}} \frac{\Gamma(v)}{\Gamma\left(\frac{v+3}{2}\right)} \frac{\rho^{\frac{v-2}{4}}}{\left(\rho^{1 / 2}+i a\right)} \times \\
& F\left(v, \frac{v-1}{2}, \frac{v+3}{2}, \frac{a+i \rho^{1 / 2}}{a-i \rho^{1 / 2}}\right) \quad \Im(\rho)<0
\end{aligned}
$$

To obtain (4.20) we have used 6.621 , (3) of ref. [9] (Here $\mathbf{F}$ is the hypergeometric function). Then we have:

$$
\begin{gathered}
F(\rho)=(4 \pi)^{\frac{v-2}{2}} i \frac{\Gamma(v)}{\Gamma\left(\frac{v+3}{2}\right)}\left\{\frac{\Theta[\mathfrak{I}(\rho)]}{\left(\rho^{1 / 2}-i a\right)} F\left(v, \frac{v-1}{2}, \frac{v+3}{2}, \frac{a-i \rho^{1 / 2}}{a+i \rho^{1 / 2}}\right)+\right. \\
\left.\frac{\Theta[-\Im(\rho)]}{\left(\rho^{1 / 2}+i a\right)} F\left(v, \frac{v-1}{2}, \frac{v+3}{2}, \frac{a+i \rho^{1 / 2}}{a-i \rho^{1 / 2}}\right)\right\}
\end{gathered}
$$

As a second example we evaluate the Fourier antitransform of $[-2 \pi i(\rho-$ $\left.\left.\mu^{2}\right)\right]^{-1}$ where $\mu$ is a complex number and $v=2 n$. Using (4.17) we have:

$$
\begin{gathered}
\hat{f}(x)=-\frac{\pi}{(2 \pi)^{\frac{v+2}{2}} \chi^{\frac{v-2}{4}}} \oint_{\Gamma} \frac{\rho^{\frac{v-2}{4}}}{2 \pi i\left(\rho-\mu^{2}\right)} \mathcal{J}_{\frac{v-2}{2}}\left(x^{1 / 2} \rho^{1 / 2}\right) d \rho= \\
\frac{\pi \mu^{\frac{v-2}{2}}}{(2 \pi)^{\frac{v+2}{2}}} \chi^{\frac{2-v}{4}} \mathcal{J}_{\frac{v-2}{2}}\left(\mu x^{1 / 2}\right)
\end{gathered}
$$

We can test the result (4.22) by transforming it. Taking into account that for $v$ even $\mathcal{J}_{\frac{v-2}{2}}=e^{\frac{i \pi(v-2)}{2}} \mathcal{J}_{\frac{2-v}{2}}$. Thus:

$$
F(\rho)=\frac{\mu^{\frac{v-2}{2}}}{4 \pi} e^{\frac{i \pi(v-2)}{2}} \rho^{\frac{2-v}{4}} \int_{0}^{\infty} \mathcal{J}_{\frac{2-v}{2}}\left(\mu x^{1 / 2}\right)\left\{\Theta[\Im(\rho)] e^{-\frac{i \pi v}{4}} \mathcal{K}_{\frac{v-2}{2}}\left(-i x^{1 / 2} \rho^{1 / 2}\right)-\right.
$$




$$
\left.\Theta[-\mathfrak{I}(\rho)] e^{\frac{i \pi v}{4}} \mathcal{K}_{\frac{v-2}{2}}\left(i x^{1 / 2} \rho^{1 / 2}\right)\right\} d x
$$

Now:

$$
\begin{gathered}
\int_{0}^{\infty} \mathcal{J}_{\frac{2-v}{2}}\left(\mu x^{1 / 2}\right) \mathcal{K}_{\frac{v-2}{2}}\left(-i x^{1 / 2} \rho^{1 / 2}\right) d x=e^{\frac{i \pi(6-v)}{4}} \mu^{\frac{2-v}{2}} \frac{\rho^{\frac{v-2}{4}}}{\rho-\mu^{2}} ; \mathfrak{I}(\rho)>0 \\
\int_{0}^{\infty} \mathcal{J}_{\frac{2-v}{2}}\left(\mu x^{1 / 2}\right) \mathcal{K}_{\frac{v-2}{2}}\left(i x^{1 / 2} \rho^{1 / 2}\right) d x=e^{-\frac{i \pi(6-v)}{4}} \mu^{\frac{2-v}{2}} \frac{\rho^{\frac{v-2}{4}}}{\rho-\mu^{2}} ; \mathfrak{I}(\rho)<0
\end{gathered}
$$

where we have used 6.576, (3) of ref. [9]. Then we have:

$$
F(\rho)=-\frac{1}{2 \pi i\left(\rho-\mu^{2}\right)}
$$

As a third example we give the Fourier transform of $\delta(x-a)$ for all $v$. Using (4.10) we obtain:

$$
\begin{gathered}
F(\rho)=\frac{(2 \pi)^{\frac{v-2}{2}}}{\rho^{\frac{v-2}{4}}} a^{\frac{v-2}{4}}\left\{\Theta[\Im(\rho)] e^{-\frac{i \pi v}{4}} \mathcal{K}_{\frac{v-2}{2}}\left(-i a^{1 / 2} \rho^{1 / 2}\right)-\right. \\
\left.\Theta[-\Im(\rho)] e^{\frac{i \pi v}{4}} \mathcal{K}_{\frac{v-2}{2}}\left(i a^{1 / 2} \rho^{1 / 2}\right)\right\}+ \\
\frac{2 \pi^{\frac{v-2}{2}}}{\Gamma\left(\frac{v-2}{2}\right) \rho^{\frac{v-2}{4}}} a^{\frac{v-2}{4}} \mathcal{S}_{\frac{v-4}{2}, \frac{v-2}{2}}\left(a^{1 / 2} \rho^{1 / 2}\right)
\end{gathered}
$$

The reader can verify that the cut of (4.26) along the negative real axis is zero. 


\section{The Fourier Transform in Minkowskian Space}

For the Minkowskian case we begin with the formula:

$$
f\left(k_{0}, k\right)=\frac{(2 \pi)^{\frac{v-1}{2}}}{k^{\frac{v-3}{2}}} \int_{-\infty}^{\infty} \int_{0}^{\infty} \hat{f}\left(x_{0}, r\right) r^{\frac{v-1}{2}} \mathcal{J}_{\frac{v-3}{2}}(k r) e^{i k_{0} x^{0}} d x^{0} d r
$$

that can be re-written as:

$$
\begin{gathered}
f\left(k_{0}^{2}-k^{2}\right)=\frac{(2 \pi)^{\frac{v-3}{2}}}{k^{\frac{v-3}{2}}} \int_{-\infty}^{\infty} \int_{-\infty}^{\infty} \int_{-\infty}^{\infty} \int_{0}^{\infty} \hat{f}(x) e^{i t\left(x-s_{0}^{2}+s^{2}\right)} s^{\frac{v-1}{2}} \mathcal{J}_{\frac{v-3}{2}}(k s) \times \\
e^{i k_{0} s^{0}} d t d x d s^{0} d s
\end{gathered}
$$

Now:

$$
\begin{gathered}
\int_{0}^{\infty} e^{i t s^{2}} s^{\frac{v-1}{2}} \\
\mathcal{J}_{\frac{v-3}{2}}(k s) d s=\frac{1}{2}\left(\frac{k}{2}\right)^{\frac{v-3}{2}}(t+i 0)^{\frac{1-v}{2}} e^{i\left[\frac{\pi}{2}\left(\frac{v-1}{2}\right)-\frac{k^{2}}{4 t}\right]} \\
\int_{-\infty}^{\infty} e^{-i t s_{0}^{2}} e^{i k_{0} s^{0}} d s^{0}=\sqrt{\pi}(t-i 0)^{-\frac{1}{2}} e^{i\left(\frac{k_{0}^{2}}{4 t}-\frac{\pi}{4}\right)}
\end{gathered}
$$

We have used 6.631, (4) and 3.462, (3) of ref. [9]. Then we obtain for (5.2):

With the results (5.3), (5.4) we obtain for (5.2):

$$
f\left(k_{0}^{2}-k^{2}\right)=\frac{(2 \pi)^{\frac{v-3}{2}}}{2^{\frac{v-1}{2}}} \sqrt{\pi} e^{\frac{i \pi(v-2)}{4}} \int_{-\infty}^{\infty} \int_{0}^{\infty} \hat{f}(x)\left[e^{i t x} e^{\frac{i\left(k_{0}^{2}-k^{2}\right)}{4 t}} t^{-\frac{v}{2}}+\right.
$$




$$
\left.e^{\frac{i \pi(2-v)}{2}} e^{-i t x} e^{-\frac{i\left(k_{0}^{2}-k^{2}\right)}{4 t}} t^{-\frac{v}{2}}\right] d x d t
$$

We can evaluate the integral in the variable $t$ :

$$
\begin{aligned}
& \int_{0}^{\infty} e^{i t x} e^{\frac{i \rho}{4 t}} t^{-\frac{v}{2}} d t=2^{\frac{v}{2}} \frac{(x+i 0)^{\frac{v-2}{4}}}{(\rho+i 0)^{\frac{v-2}{4}}} \mathcal{K}_{\frac{v-2}{2}}\left[-i(x+i 0)^{1 / 2}(\rho+i 0)^{1 / 2}\right] \\
& \int_{0}^{\infty} e^{-i t x} e^{-\frac{i \rho}{4 t}} t^{-\frac{v}{2}} d t=2^{\frac{v}{2}} \frac{(x-i 0)^{\frac{v-2}{4}}}{(\rho-i 0)^{\frac{v-2}{4}}} \mathcal{K}_{\frac{v-2}{2}}\left[i(x-i 0)^{1 / 2}(\rho-i 0)^{1 / 2}\right]
\end{aligned}
$$

where $\rho=k_{0}^{2}-k^{2}$ (Here we have used 3.471, (9) of ref. [9]). Thus (5.5]) transforms into:

$$
\begin{gathered}
f(\rho)=(2 \pi)^{\frac{v-2}{2}} \int_{-\infty}^{\infty} \hat{f}(x)\left\{e^{\frac{i \pi(v-2)}{4}} \frac{(x+i 0)^{\frac{v-2}{4}}}{(\rho+i 0)^{\frac{v-2}{4}}} \mathcal{K}_{\frac{v-2}{2}}\left[-i(x+i 0)^{1 / 2}(\rho+i 0)^{1 / 2}\right]+\right. \\
\left.+e^{\frac{i \pi(2-v)}{4}} \frac{(x-i 0)^{\frac{v-2}{4}}}{(\rho-i 0)^{\frac{v-2}{4}}} \mathcal{K}_{\frac{v-2}{2}}\left[i(x-i 0)^{1 / 2}(\rho-i 0)^{1 / 2}\right]\right\} d x
\end{gathered}
$$

The corresponding inversion formula is then given by:

$$
\begin{gathered}
\hat{f}(x)=\frac{1}{(2 \pi)^{\frac{v+2}{2}}} \int_{-\infty}^{\infty} f(\rho)\left\{e^{\frac{i \pi(v-2)}{4}} \frac{(\rho+i 0)^{\frac{v-2}{4}}}{(x+i 0)^{\frac{v-2}{4}}} \mathcal{K}_{\frac{v-2}{2}}\left[-i(x+i 0)^{1 / 2}(\rho+i 0)^{1 / 2}\right]+\right. \\
\left.+e^{\frac{i \pi(2-v)}{4}} \frac{(\rho-i 0)^{\frac{v-2}{4}}}{(x-i 0)^{\frac{v-2}{4}}} \mathcal{K}_{\frac{v-2}{2}}\left[i(x-i 0)^{1 / 2}(\rho-i 0)^{1 / 2}\right]\right\} d \rho
\end{gathered}
$$


Formula (5.7) is the generalization of Bochner's formula (4.1) to the Minkowskian Space.

In this case the extension as ultradistribution of $f(\rho)$ to the complex $\rho$-plane is immediate:

$$
\begin{gathered}
F(\rho)=(2 \pi)^{\frac{v-2}{2}} \int_{-\infty}^{\infty} \hat{f}(x)\left\{\Theta[\Im(\rho)] e^{\frac{\mathfrak{i} \pi(v-2)}{4}} \frac{(x+i 0)^{\frac{v-2}{4}}}{\rho^{\frac{v-2}{4}}} \mathcal{K}_{\frac{v-2}{2}}\left[-\mathfrak{i}(x+\mathfrak{i} 0)^{1 / 2} \rho^{1 / 2}\right]-\right. \\
\left.\Theta[-\Im(\rho)] e^{\frac{i \pi(2-v)}{4}} \frac{(x-\mathfrak{i} 0)^{\frac{v-2}{4}}}{\rho^{\frac{v-2}{4}}} \mathcal{K}_{\frac{v-2}{2}}\left[\mathfrak{i}(x-\mathfrak{i} 0)^{1 / 2} \rho^{1 / 2}\right]\right\} d x
\end{gathered}
$$

Here we have taken $\rho=\gamma+i \sigma$ and

$$
\rho^{1 / 2}=\sqrt{\frac{\gamma+\sqrt{\gamma^{2}+\sigma^{2}}}{2}}+i \operatorname{Sgn}(\sigma) \sqrt{\frac{-\gamma+\sqrt{\gamma^{2}+\sigma^{2}}}{2}}
$$

Now we can define a Lorentz invariant tempered ultradistribution as the Fourier transform of a Lorentz invariant distribution of exponential type. Note that a Lorentz invariant tempered ultradistribution is not necessarily explicitly Lorentz invariant. When $\hat{f}$ is a Lorentz invariant distribution of exponential type, we can use (5.9) or to adopt the following treatment: starting from

$$
\iiint_{-\infty}^{\infty} \int f(\rho) \phi\left(\rho, k^{0}\right) d^{4} k=(2 \pi)^{v} \iiint \int_{-\infty}^{\infty} \int_{f} \hat{f}(x) \hat{\phi}\left(x, x^{0}\right) d^{4} x
$$


can be deduced the equality:

$$
\begin{gathered}
\int_{-\infty}^{\infty} f(\rho) \phi\left(\rho, k^{0}\right)\left(k_{0}^{2}-\rho\right)_{+}^{\frac{v-3}{2}} d \rho d k^{0}= \\
\iint_{-\infty}^{\infty} \hat{f}(x) \hat{\phi}\left(x, x^{0}\right)\left(x-x_{0}^{2}\right)_{+}^{\frac{v-3}{2}} d x d x^{0}
\end{gathered}
$$

Let $g(t)$ defined as:

$$
\hat{g}(t)=\frac{1}{(2 \pi)^{v}} \int_{-\infty}^{\infty} f(\rho) e^{-i \rho t} d \rho
$$

Then:

$$
F(\rho)=\Theta[\Im(\rho)] \int_{0}^{\infty} \hat{g}(t) e^{i \rho t} d t-\Theta[-\mathfrak{I}(\rho)] \int_{-\infty}^{0} \hat{g}(t) e^{i \rho t} d t
$$

or if we use Dirac's formula

$$
F(\rho)=\frac{1}{2 \pi i} \int_{-\infty}^{\infty} \frac{f(t)}{t-\rho} d t
$$

The inverse of the Fourier transform can also be evaluated in the following way: we define:

$$
\begin{aligned}
\hat{G}(x, \Lambda) & =\frac{1}{(2 \pi)^{\frac{v+2}{2}}} \oint_{\Gamma} F(\rho)\left\{e^{\frac{i \pi(v-2)}{4}} \frac{(\rho+\Lambda)^{\frac{v-2}{4}}}{(x+i 0)^{\frac{v-2}{4}}} \mathcal{K}_{\frac{v-2}{2}}\left[-i(x+i 0)^{1 / 2}(\rho+\Lambda)^{1 / 2}\right]+\right. \\
& \left.+e^{\frac{i \pi(2-v)}{4}} \frac{(\rho-\Lambda)^{\frac{v-2}{4}}}{(x-i 0)^{\frac{v-2}{4}}} \mathcal{K}_{\frac{v-2}{2}}\left[i(x-i 0)^{1 / 2}(\rho-\Lambda)^{1 / 2}\right]\right\} d \rho
\end{aligned}
$$

then

$$
\hat{f}(x)=\hat{G}\left(x, i 0^{+}\right)
$$




\section{Examples}

As a first example we consider the Fourier transform of the function $e^{a \sqrt{\left|x_{0}^{2}-r^{2}\right|}}$ where a is a complex number. The Fourier transform is:

$$
\begin{aligned}
F(\rho)= & (2 \pi)^{\frac{v-2}{2}} \int_{-\infty}^{\infty} e^{|x|^{\frac{1}{2}}}\left\{\Theta[\mathfrak{I}(\rho)] e^{\frac{i \pi(v-2)}{4}} \frac{(x+i 0)^{\frac{v-2}{4}}}{\rho^{\frac{v-2}{4}}} \mathcal{K}_{\frac{v-2}{2}}\left[-i(x+i 0)^{1 / 2} \rho^{1 / 2}\right]-\right. \\
& \left.\Theta[-\mathfrak{I}(\rho)] e^{\frac{i \pi(2-v)}{4}} \frac{(x-i 0)^{\frac{v-2}{4}}}{\rho^{\frac{v-2}{4}}} \mathcal{K}_{\frac{v-2}{2}}\left[i(x-i 0)^{1 / 2} \rho^{1 / 2}\right]\right\} d x
\end{aligned}
$$

Now:

$$
\begin{aligned}
& e^{\frac{i \pi(v-2)}{4}} \int_{-\infty}^{\infty} e^{a|x|^{\frac{1}{2}}}(x+i 0)^{\frac{v-2}{4}} \mathcal{K}_{\frac{v-2}{2}}\left[-i(x+i 0)^{1 / 2} \rho^{1 / 2}\right]= \\
& 2^{\frac{v}{2}} \sqrt{\pi} \frac{\Gamma(v)}{\Gamma\left(\frac{v+3}{2}\right)} \frac{e^{\frac{i \pi v}{2}}}{\left(\rho^{1 / 2}-i a\right)^{v}} F\left(v, \frac{v-1}{2}, \frac{v+3}{2}, \frac{a-i \rho^{1 / 2}}{a+i \rho^{1 / 2}}\right)- \\
& 2^{\frac{v}{2}} \sqrt{\pi} \frac{\Gamma(v)}{\Gamma\left(\frac{v+3}{2}\right)} \frac{e^{\frac{i \pi v}{2}}}{\left(\rho^{1 / 2}+a\right)^{v}} F\left(v, \frac{v-1}{2}, \frac{v+3}{2}, \frac{a+\rho^{1 / 2}}{a-\rho^{1 / 2}}\right) \quad \mathfrak{I}(\rho)>0 \\
& e^{\frac{i \pi(2-v)}{4}} \int_{-\infty}^{\infty} e^{a|x|^{\frac{1}{2}}}(x-i 0)^{\frac{v-2}{4}} \mathcal{K}_{\frac{v-2}{2}}\left[i(x-i 0)^{1 / 2} \rho^{1 / 2}\right]= \\
& 2^{\frac{v}{2}} \sqrt{\pi} \frac{\Gamma(v)}{\Gamma\left(\frac{v+3}{2}\right)} \frac{e^{-\frac{i \pi v}{2}}}{\left(\rho^{1 / 2}+i a\right)^{v}} F\left(v, \frac{v-1}{2}, \frac{v+3}{2}, \frac{a+i \rho^{1 / 2}}{a-i \rho^{1 / 2}}\right)- \\
& 2^{\frac{v}{2}} \sqrt{\pi} \frac{\Gamma(v)}{\Gamma\left(\frac{v+3}{2}\right)} \frac{e^{\frac{i \pi v}{2}}}{\left(\rho^{1 / 2}+a\right)^{v}} F\left(v, \frac{v-1}{2}, \frac{v+3}{2}, \frac{a+\rho^{1 / 2}}{a-\rho^{1 / 2}}\right) \quad \mathfrak{I}(\rho)<0
\end{aligned}
$$


To obtain (5.17) and (5.18) we have used 6.621,(3) of ref. [9]. With these results we have:

$$
\begin{gathered}
F(\rho)=(4 \pi)^{\frac{v-1}{2}} \frac{\Gamma(v)}{\Gamma\left(\frac{v+3}{2}\right)}\left\{\Theta [ \Im ( \rho ) ] e ^ { \frac { i \pi v } { 2 } } \left[\frac{F\left(v, \frac{v-1}{2}, \frac{v+3}{2}, \frac{a-i \rho^{1 / 2}}{a+i \rho^{1 / 2}}\right)}{\left(\rho^{1 / 2}-i a\right)^{v}}-\right.\right. \\
\left.\frac{F\left(v, \frac{v-1}{2}, \frac{v+3}{2}, \frac{a+\rho^{1 / 2}}{a-\rho^{1 / 2}}\right)}{\left(\rho^{1 / 2}+a\right)^{v}}\right]-\Theta[-\Im(\rho)] e^{-\frac{i \pi v}{2}}\left[\frac{F\left(v, \frac{v-1}{2}, \frac{v+3}{2}, \frac{a+i \rho^{1 / 2}}{a-i \rho^{1 / 2}}\right)}{\left(\rho^{1 / 2}+i a\right)^{v}}-\right. \\
\left.\left.\frac{F\left(v, \frac{v-1}{2}, \frac{v+3}{2}, \frac{a+\rho^{1 / 2}}{a-\rho^{1 / 2}}\right)}{\left(\rho^{1 / 2}+a\right)^{v}}\right]\right\}
\end{gathered}
$$

As a second example we evaluate the Fourier transform of the complex mass Wheeler's propagator.

$$
\mathcal{w}_{\mu}(x)=-\frac{i \pi}{2} \frac{\mu^{\frac{v-2}{2}}}{(2 \pi)^{\frac{v}{2}}} \chi_{+}^{\frac{2-v}{4}} \mathcal{J}_{\frac{2-v}{2}}\left(\mu x_{+}^{1 / 2}\right)
$$

Then according to (5.9)

$$
\begin{gathered}
\mathcal{W}_{\mu}(\rho)=-\frac{i(\mu)^{\frac{v-2}{4}}}{4} \int_{0}^{\infty} \mathcal{J}_{\frac{2-v}{2}}\left(\mu x^{1 / 2}\right)\left[\Theta[\Im(\rho)] \frac{e^{\frac{i \pi(v-2)}{4}}}{\rho^{\frac{v-2}{4}}} \mathcal{K}_{\frac{v-2}{2}}\left(-i x^{1 / 2} \rho^{1 / 2}\right)-\right. \\
\left.\Theta[-\Im(\rho)] \frac{e^{\frac{i \pi(2-v)}{4}}}{\rho^{\frac{v-2}{4}}} \mathcal{K}_{\frac{v-2}{2}}\left(i x^{1 / 2} \rho^{1 / 2}\right)\right] d x
\end{gathered}
$$

Taking into account that (See 6.576, (3), ref. 9]):

$$
\int_{0}^{\infty} \mathcal{J}_{\frac{2-v}{2}}\left(\mu x^{1 / 2}\right) \mathcal{K}_{\frac{v-2}{2}}\left(-i x^{1 / 2} \rho^{1 / 2}\right) d x=2 \mu^{\frac{2-v}{2}} e^{\frac{i \pi(6-v)}{4}} \frac{\rho^{\frac{v-2}{4}}}{\rho-\mu^{2}} \mathfrak{I}(\rho)>0
$$




$$
\int_{0}^{\infty} \mathcal{J}_{\frac{2-v}{2}}\left(\mu x^{1 / 2}\right) \mathcal{K}_{\frac{v-2}{2}}\left(i x^{1 / 2} \rho^{1 / 2}\right) d x=2 \mu^{\frac{2-v}{2}} e^{\frac{i \pi(v-6)}{4}} \frac{\rho^{\frac{v-2}{4}}}{\rho-\mu^{2}} \mathfrak{I}(\rho)<0
$$

we obtain:

$$
\mathcal{W}_{\mu}(\rho)=\frac{i}{2} \frac{\operatorname{Sgn}[\Im(\rho)]}{\rho-\mu^{2}}
$$

As a third example we evaluate the transform of $\delta\left(x_{0}^{2}-r^{2}\right)$. From (5.12) we obtain:

$$
\int_{-\infty}^{\infty} f(\rho) \phi\left(\rho, k^{0}\right)\left(k_{0}^{2}-\rho\right)_{+}^{\frac{v-3}{2}} d \rho d k^{0}=(2 \pi)^{v} \int_{-\infty}^{\infty} \phi\left(0, x^{0}\right)\left|x^{0}\right|^{v-3} d x^{0}
$$

According to (5.1) we can write:

$$
\begin{gathered}
\hat{\phi}\left(x, x^{0}\right)=2^{-1}(2 \pi)^{-\frac{v+1}{2}}\left(x_{0}^{2}-x\right)_{+}^{\frac{3-v}{4}} \int_{-\infty}^{\infty} \int_{\infty} \phi\left(\rho, k^{0}\right) \mathcal{J}_{\frac{v-3}{2}}\left[\left(x_{0}^{2}-x\right)_{+}^{1 / 2}\left(k_{0}^{2}-\rho\right)_{+}^{1 / 2}\right] \times \\
\left(k_{0}^{2}-\rho\right)_{+}^{\frac{v-3}{4}} e^{i k_{0} x^{0}} d k^{0} d \rho
\end{gathered}
$$

and consequently:

$$
\begin{gathered}
\hat{\phi}\left(0, x^{\mathcal{O}}\right)=2^{-1}(2 \pi)^{-\frac{v+1}{2}}\left|\chi^{\mathcal{O}}\right|^{\frac{3-v}{4}} \int_{-\infty}^{\infty} \int_{\infty} \phi\left(\rho, k^{\mathcal{O}}\right) \mathcal{J}_{\frac{v-3}{2}}\left[\left|x^{\mathcal{O}}\right|^{1 / 2}\left(k_{0}^{2}-\rho\right)_{+}^{1 / 2}\right] \times \\
\left(k_{0}^{2}-\rho\right)_{+}^{\frac{v-3}{4}} e^{i k_{0} x^{\mathcal{O}}} d k^{\mathcal{O}} \mathrm{d} \rho
\end{gathered}
$$


Then

$$
\begin{gathered}
(2 \pi)^{v} \int_{-\infty}^{\infty} \phi\left(0, x^{0}\right)\left|x^{0}\right|^{v-3} \mathrm{~d} x^{0}=2^{-1}(2 \pi)^{\frac{v-1}{2}} \int_{-\infty}^{\infty} \phi\left(\rho, \mathrm{k}^{0}\right)\left(k_{0}^{2}-\rho\right)_{+}^{\frac{v-3}{4}}\left[\int_{-\infty}^{\infty}\left|x^{0}\right|^{\frac{v-3}{2}} x\right. \\
\left.\mathcal{J}_{\frac{v-3}{2}}\left[\left|x^{0}\right|^{1 / 2}\left(k_{0}^{2}-\rho\right)_{+}^{1 / 2}\right] e^{i k_{0} x^{0}} \mathrm{~d} x^{0}\right] \mathrm{d} k^{0} \mathrm{~d} \rho
\end{gathered}
$$

But

$$
\begin{gathered}
\int_{-\infty}^{\infty}\left|x^{0}\right|^{\frac{v-3}{2}} \mathcal{J}_{\frac{v-3}{2}}\left[\left|x^{0}\right|^{1 / 2}\left(k_{0}^{2}-\rho\right)_{+}^{1 / 2}\right] e^{i k_{0} x^{0}} d x^{0}= \\
\frac{2^{\frac{v-3}{2}}}{\sqrt{\pi}} \Gamma\left(\frac{v-2}{2}\right)\left[e^{\frac{i \pi(v-2)}{2}}(\rho+i 0)^{\frac{2-v}{2}}+e^{\frac{i \pi(2-v)}{2}}(\rho-i 0)^{\frac{2-v}{2}}\right]
\end{gathered}
$$

(See 6.623, (1),ref $[9]$ )

from which we deduce that

$$
f(\rho)=\frac{(4 \pi)^{\frac{v-2}{2}}}{2} \Gamma\left(\frac{v-2}{2}\right)\left[\frac{e^{\frac{i \pi(v-2)}{2}}}{(\rho+i 0)^{\frac{v-2}{2}}}+\frac{e^{\frac{i \pi(2-v)}{2}}}{(\rho-i 0)^{\frac{v-2}{2}}}\right]
$$

Using then $[(15.13),(15.14)]$ or $(\underline{5.15})$, the corresponding ultradistribution is:

$$
F(\rho)=2^{-1}(4 \pi)^{\frac{v-2}{2}} \Gamma\left(\frac{v-2}{2}\right) \operatorname{Sgn}[\Im(\rho)](-\rho)^{\frac{2-v}{2}}
$$

We proceed now to the calculation of the convolution of two spherically symmetric tempered ultradistributions. 


\section{The Convolution in Euclidean Space}

The expression for the convolution of two spherically symmetric functions was deduced in ref.[13] $(h(k)=(f * g)(k))$ :

$$
\begin{gathered}
h(k)=\frac{2^{4-v} \pi^{\frac{v-1}{2}}}{\Gamma\left(\frac{v-1}{2}\right) k^{v-2}} \int_{0}^{\infty} f\left(k_{1}\right) g\left(k_{2}\right) \times \\
{\left[4 k_{1}^{2} k_{2}^{2}-\left(k^{2}-k_{1}^{2}-k_{2}^{2}\right)^{2}\right]_{+}^{\frac{v-3}{2}} k_{1} k_{2} d k_{1} d k_{2}}
\end{gathered}
$$

and with the change of variables $\rho=k^{2}, \rho_{1}=k_{1}^{2}, \rho_{2}=k_{2}^{2}$ takes the form:

$$
\begin{aligned}
& h(\rho)=\frac{2^{2-v} \pi^{\frac{v-1}{2}}}{\Gamma\left(\frac{v-1}{2}\right) \rho^{\frac{v-2}{2}}} \int_{0}^{\infty} f\left(\rho_{1}\right) g\left(\rho_{2}\right) \times \\
& {\left[4 \rho_{1} \rho_{2}-\left(\rho-\rho_{1}-\rho_{2}\right)^{2}\right]_{+}^{\frac{v-3}{2}} d \rho_{1} d \rho_{2}}
\end{aligned}
$$

In particular when $v=4$ is:

$$
h(\rho)=\frac{\pi}{2 \rho} \iint_{0}^{\infty} f\left(\rho_{1}\right) g\left(\rho_{2}\right)\left[4 \rho_{1} \rho_{2}-\left(\rho-\rho_{1}-\rho_{2}\right)^{2}\right]_{+}^{\frac{1}{2}} d \rho_{1} d \rho_{2}
$$

$h(\rho)$ can be extended to complex plane as ultradistribution thus generalizing the procedure of ref. 13]. According to (4.12) we can write:

$$
\hat{\mathrm{f}}(x) \hat{\mathrm{g}}(x)=\frac{\pi^{2}}{(2 \pi)^{6} \chi} \oint_{\Gamma_{1}} \oint_{\Gamma_{2}} F\left(\rho_{1}\right) G\left(\rho_{2}\right) \rho_{1}^{1 / 2} \rho_{2}^{1 / 2} \mathcal{J}_{1}\left(x^{1 / 2} \rho_{1}^{1 / 2}\right) \mathcal{J}_{1}\left(x^{1 / 2} \rho_{2}^{1 / 2}\right) \mathrm{d} \rho_{1} \mathrm{~d} \rho_{2}
$$


and Fourier transforming:

$$
\begin{gathered}
\mathcal{F}\{\hat{f}(x) \widehat{g}(x)\}(\rho)=\frac{-\pi^{2}}{(2 \pi)^{5} \rho^{1 / 2}} \oint_{\Gamma_{1}} \oint_{\Gamma_{2}} F\left(\rho_{1}\right) G\left(\rho_{2}\right) \rho_{1}^{1 / 2} \rho_{2}^{1 / 2}\left\{\int_{0}^{\infty} x^{-1 / 2} \mathcal{J}_{1}\left(x^{1 / 2} \rho_{1}^{1 / 2}\right) \mathcal{J}_{1}\left(x^{1 / 2} \rho_{2}^{1 / 2}\right)\right. \\
\left.\left[\Theta[\Im(\rho)] \mathcal{K}_{1}\left(-i x^{1 / 2} \rho^{1 / 2}\right)-\Theta[-\Im(\rho)] \mathcal{K}_{1}\left(i x^{1 / 2} \rho^{1 / 2}\right)\right] d x\right\} d \rho_{1} d \rho_{2}
\end{gathered}
$$

The x-integration can be performed with the result:

$$
\begin{aligned}
\int_{0}^{\infty} \mathcal{J}_{1}\left(x^{1 / 2} \rho_{1}^{1 / 2}\right) \mathcal{J}_{1}\left(x^{1 / 2} \rho_{2}^{1 / 2}\right) \mathcal{K}_{1}\left(-i x^{1 / 2} \rho^{1 / 2}\right) d x= \\
-i\left(\rho \rho_{1} \rho_{2}\right)^{-1}\left[\rho-\rho_{1}-\rho_{2}-\sqrt{\left(\rho-\rho_{1}-\rho_{2}\right)^{2}-4 \rho_{1} \rho_{2}}\right] \quad \mathfrak{I}(\rho)>0 \\
\int_{0}^{\infty} \mathcal{J}_{1}\left(x^{1 / 2} \rho_{1}^{1 / 2}\right) \mathcal{J}_{1}\left(x^{1 / 2} \rho_{2}^{1 / 2}\right) \mathcal{K}_{1}\left(i x^{1 / 2} \rho^{1 / 2}\right) d x= \\
i\left(\rho \rho_{1} \rho_{2}\right)^{-1}\left[\rho-\rho_{1}-\rho_{2}-\sqrt{\left(\rho-\rho_{1}-\rho_{2}\right)^{2}-4 \rho_{1} \rho_{2}}\right] \quad \mathfrak{I}(\rho)<0
\end{aligned}
$$

where we have used $6.578,2$ of [9] and (7) pag. 238 of [12]. Thus

$$
\begin{gathered}
H(\rho)=\frac{i \pi}{4 \rho} \oint_{\Gamma_{1} \oint_{2}} F\left(\rho_{1}\right) G\left(\rho_{2}\right) \times \\
{\left[\rho-\rho_{1}-\rho_{2}-\sqrt{\left(\rho-\rho_{1}-\rho_{2}\right)^{2}-4 \rho_{1} \rho_{2}}\right] d \rho_{1} d \rho_{2}}
\end{gathered}
$$


$|\mathfrak{I}(\rho)|>\left|\mathfrak{I}\left(\rho_{1}\right)\right|+\left|\mathfrak{I}\left(\rho_{2}\right)\right|$

In ref. 3. we have defined and shown the existence of the convolution product between to arbitrary one dimensional tempered ultradistributions. Analogously for spherically symmetric ultradistributions we now define:

$$
\begin{gathered}
H_{\lambda}(\rho)=\frac{i \pi}{4 \rho} \oint_{\Gamma_{1}} \oint_{\Gamma_{2}} F\left(\rho_{1}\right) G\left(\rho_{2}\right) \rho_{1}^{\lambda} \rho_{2}^{\lambda} \times \\
{\left[\rho-\rho_{1}-\rho_{2}-\sqrt{\left(\rho-\rho_{1}-\rho_{2}\right)^{2}-4 \rho_{1} \rho_{2}}\right] d \rho_{1} d \rho_{2}}
\end{gathered}
$$

Let $\mathfrak{b}$ be a vertical band contained in the complex $\lambda$-plane $\mathfrak{7}$. Integral (6.9) is an analytic function of $\lambda$ defined in the domain $\mathbf{3}$. Moreover, it is bounded by a power of $|\rho|$. Then, according to the method of ref. [8], $\mathrm{H}_{\lambda}$ can be analytically continued to other parts of $\mathfrak{x}$. In particular near the origin we have the Laurent expansion:

$$
H_{\lambda}(\rho)=\sum_{n=-m}^{\infty} H^{(n)}(\rho) \lambda^{n}
$$

We now define the convolution product as the $\lambda$-independent term of (6.10):

$$
H(\rho)=H^{(0)}(\rho)
$$

The proof that $H(\rho)$ is a Tempered Ultradistribution is similar to the one given in ref. 3. for the one-dimensional case. The Fourier antitransform of 
(6.11) defines the product of two distributions of exponential type. Let $\hat{H}_{\lambda}(x)$ be the Fourier antitransform of $\mathrm{H}_{\lambda}(\rho)$ :

$$
\hat{\mathrm{H}}_{\lambda}(x)=\sum_{n=-m}^{\infty} \hat{\mathrm{H}}^{(\mathrm{n})}(x) \lambda^{n}
$$

If we define:

$$
\begin{gathered}
\hat{f}_{\lambda}(x)=\mathcal{F}^{-1}\left\{\rho^{\lambda} F(\rho)\right\} \\
\hat{g}_{\lambda}(x)=\mathcal{F}^{-1}\left\{\rho^{\lambda} G(\rho)\right\}
\end{gathered}
$$

then

$$
\hat{\mathrm{H}}_{\lambda}(x)=(2 \pi)^{4} \hat{\mathrm{f}}_{\lambda}(x) \hat{g}_{\lambda}(x)
$$

and taking into account the Laurent developments of $\hat{f}$ and $\hat{g}$ :

$$
\begin{gathered}
\hat{f}_{\lambda}(x)=\sum_{n=-m_{f}}^{\infty} \hat{f}^{(n)}(x) \lambda^{n} \\
\hat{g}_{\lambda}(x)=\sum_{n=-m_{g}}^{\infty} \hat{g}^{(n)}(x) \lambda^{n}
\end{gathered}
$$

we can write:

$$
\sum_{n=-m}^{\infty} \hat{H}^{(n)}(x) \lambda^{n}=(2 \pi)^{4} \sum_{n=-m}^{\infty}\left(\sum_{k=-m_{f}}^{n+m_{g}} \hat{f}^{(k)}(x) \hat{g}^{(n-k)}(x)\right) \lambda^{n}
$$


$\left(m=m_{f}+m_{g}\right)$

and as a consequence:

$$
\hat{H}^{(0)}(x)=\sum_{k=-m_{f}}^{m_{g}} \hat{f}^{(k)}(x) \hat{g}^{(-k)}(x)
$$

We will give now some examples of the use of (6.11) and (6.17).

\section{Examples}

As a first example we evaluate the convolution of two Dirac's delta of complex mass:

$$
\left(\delta\left(\rho-\mu^{2}\right)=-\frac{1}{2 \pi i\left(\rho-\mu^{2}\right)}\right)
$$

According to (6.9), 6.10), 6.11) we have:

$$
\delta\left(\rho-\mu_{1}^{2}\right) * \delta\left(\rho-\mu_{2}^{2}\right)=\frac{i \pi}{4 \rho}\left[\rho-\mu_{1}^{2}-\mu_{2}^{2}-\sqrt{\left(\rho-\mu_{1}^{2}-\mu_{2}^{2}\right)^{2}-4 \mu_{1}^{2} \mu_{2}^{2}}\right]
$$

As an ultradistribution only the term containing the square root is different from zero (cf.(4.11) $)$. We then have:

$$
\delta\left(\rho-\mu_{1}^{2}\right) * \delta\left(\rho-\mu_{2}^{2}\right)=-\frac{i \pi}{4 \rho} \sqrt{\left(\rho-\mu_{1}^{2}-\mu_{2}^{2}\right)^{2}-4 \mu_{1}^{2} \mu_{2}^{2}}
$$

When $\mu_{1}=\mu_{2}=\mathrm{m}$ ( $\mathrm{m}$ real) we obtain:

$$
\delta\left(\rho-m^{2}\right) * \delta\left(\rho-m^{2}\right)=-\frac{i \pi}{4 \rho^{1 / 2}} \sqrt{\rho-4 m^{2}}
$$


As a second example we evaluate the convolution of two massless Feynman's propagators. We have:

$$
\begin{gathered}
f(\rho)=\frac{1}{\rho} \\
F(\rho)=-\frac{1}{2 \pi i \rho} \ln (-\rho) \\
F_{\lambda}(\rho)=-\frac{1}{2 \pi i} \rho^{\lambda-1} \ln (-\rho) \\
\hat{f}_{\lambda}(x)=\frac{1}{8 \pi^{2} \chi^{1 / 2}} \oint_{\Gamma}\left(-\frac{1}{2 \pi i} \rho^{\lambda-1} \ln (-\rho)\right) \rho^{1 / 2} \mathcal{J}_{1}\left(\chi^{1 / 2} \rho^{1 / 2}\right) d \rho= \\
\frac{2^{2 \lambda} \Gamma(1+\lambda)}{4 \pi^{2} \Gamma(1-\lambda)} x^{-\lambda-1}-e^{i \pi \lambda} \sin (\pi \lambda) \frac{2^{2 \lambda} \Gamma(1+\lambda)}{4 \pi^{2} \Gamma(1-\lambda)} x^{-\lambda-1}[i \pi+ \\
2 \ln (2)+\psi(1+\lambda)+\psi(1-\lambda)-\ln (x)]
\end{gathered}
$$

where $\psi(z)=\Gamma^{\prime}(z) / \Gamma(z)$

From 6.20) we have

$$
\hat{f}_{\lambda}(x)=(2 \pi)^{-2} \chi^{-1}+S_{\lambda}(x)
$$

with

$$
\lim _{\lambda \rightarrow 0} S_{\lambda}(x)=0
$$

Then

$$
\hat{f}_{\lambda}^{2}(x)=(2 \pi)^{-4} x^{-2}+T_{\lambda}(x)
$$


with

$$
\lim _{\lambda \rightarrow 0} T_{\lambda}(x)=0
$$

As a consequence

$$
\hat{f}^{2}(x)=(2 \pi)^{-4} x^{-2}
$$

Taking into account that

$$
\mathcal{F}\left\{x^{-2}\right\}=-\pi^{2} \ln (\rho)
$$

we obtain

$$
\frac{1}{\rho} * \frac{1}{\rho}=-\pi^{2} \ln (\rho)
$$

\section{The Convolution in Minkowskian space}

In this section we deduce the formula for the convolution of two Lorentz invariant functions and then we consider the central topic of this paper, i.e: the convolution of two Lorentz invariant tempered ultradistributions. 


\subsection{The generalization of Dimensional Regularization in Configuration Space to the Minkowskian Space}

The convolution of two Lorentz invariant functions is given by:

$$
\{f * g\}\left(p_{\mu}^{2}\right)=\int_{-\infty}^{\infty} \cdots \int_{-\infty}^{\infty} f\left[\left(p_{\mu}-k_{\mu}\right)^{2}\right] g\left(k_{\mu}^{2}\right) d^{v} k
$$

and can be re-written as

$$
\int_{-\infty}^{\infty} \cdots \int_{-\infty}^{\infty} f\left(\eta_{1}\right) g\left(\eta_{2}\right) \delta\left[\eta_{1}-\left(p_{\mu}-k_{\mu}\right)^{2}\right] \delta\left(\eta_{2}-k_{\mu}^{2}\right) d \eta_{1} d \eta_{2} d^{v} k
$$

We select the axis of coordinates in a way that the spatial component of $p_{\mu}$,

$\vec{p}$ coincides with the first spatial coordinate $\left(p_{\mu}^{2}=p_{0}^{2}-p_{1}^{2}\right)$. Then we have:

$$
\frac{\pi^{\frac{v-2}{2}}}{2\left|p_{0}\right|} \iiint_{-\infty}^{\infty} \frac{f\left(\eta_{1}\right) g\left(\eta_{2}\right)}{\Gamma\left(\frac{v-2}{2}\right)}\left[\frac{\left(p_{\mu}^{2}-\eta_{1}+\eta_{2}+2 p_{1} k_{1}\right)^{2}}{4 p_{0}^{2}}-k_{1}^{2}-\eta_{2}\right]^{\frac{v-4}{2}} d \eta_{1} d \eta_{2} d k_{1}
$$

Using:

$$
x_{+}^{\frac{v-4}{2}}=\frac{\Gamma\left(\frac{v-2}{2}\right) e^{i \pi\left(\frac{2-v}{4}\right)}}{2 \pi} \int_{-\infty}^{\infty}(t-i 0)^{\frac{3-v}{2}} e^{i t x} d t
$$

with

$$
x=-4 p_{\mu}^{2} k_{1}^{2}+4 p_{1} k_{1}\left(p_{\mu}^{2}-\eta_{1}+\eta_{2}\right)+\left(p_{\mu}^{2}-\eta_{1}+\eta_{2}\right)^{2}-4 p_{o}^{2} \eta_{2}
$$

we can evaluate the integral in the variable $k_{1}$ using $2.462(1)$ of ref. [9]. The result is:

$$
\sqrt{2 \pi}\left[i\left(8 t p_{\mu}^{2}-i 0\right)\right]^{-\frac{1}{2}} e^{\frac{i t p_{1}^{2}\left(p_{\mu}^{2}-\eta_{1}+\eta_{2}\right)}{p_{\mu}^{2}}}
$$


We can now perform the $t$ integration:

$$
I=\lim _{\epsilon \rightarrow 0} \frac{\Gamma\left(\frac{v-2}{2}\right) e^{\frac{i \pi(1-v)}{4}}}{4 \sqrt{\pi}} \int_{-\infty}^{\infty}(t-i \epsilon)^{\frac{2-v}{2}}\left(t p_{\mu}^{2}-i \epsilon\right)^{-\frac{1}{2}} e^{\frac{i t p_{0}^{2}\left[\left(p_{\mu}^{2}-\eta_{1}+\eta_{2}\right)^{2}-4 p_{\mu}^{2} \eta_{2}\right]}{p_{\mu}^{2}}} d t
$$

Formula (7.7) is defined for $v=2 \mathrm{n}$. In this case (7.7) is proportional to the derivative of the same order of the Dirac's formula for

$$
\left(t p_{\mu}^{2}-i 0\right)^{-\frac{1}{2}} e^{\frac{i t p_{0}^{2}\left[\left(p_{\mu}^{2}-\eta_{1}+\eta_{2}\right)^{2}-4 p_{\mu}^{2} \eta_{2}\right]}{p_{\mu}^{2}}}
$$

with $z=i \epsilon$. Thus we have

$$
\begin{gathered}
I=\frac{\Gamma\left(\frac{v-2}{2}\right) e^{\frac{i \pi(1-v)}{4}}}{4 \sqrt{\pi}} \int_{-\infty}^{\infty}\left(p_{\mu}^{2}-i 0\right)^{-\frac{1}{2}} t_{+}^{\frac{1-v}{2}} e^{\frac{i t p_{0}^{2}\left[\left(p_{\mu}^{2}-\eta_{1}+\eta_{2}\right)^{2}-4 p_{\mu}^{2} \eta_{2}\right]}{p_{\mu}^{2}}}+ \\
\left(p_{\mu}^{2}+i 0\right)^{-\frac{1}{2}} t_{+}^{\frac{1-v}{2}} e^{-\frac{i t p_{0}^{2}\left[\left(p_{\mu}^{2}-\eta_{1}+\eta_{2}\right)^{2}-4 p_{\mu}^{2} \eta_{2}\right]}{p_{\mu}^{2}}} d t
\end{gathered}
$$

The result of (7.8) is immediate (is a Fourier transform). We consider first the case $v \neq 2 n+1$ :

$$
\begin{gathered}
I=\frac{e^{\frac{i \pi(2-v)}{2}}}{4 \sqrt{\pi}} \Gamma\left(\frac{v-2}{2}\right) \Gamma\left(\frac{3-v}{2}\right)\left|p_{0}\right|^{v-3} \times \\
\left\{\left(p_{\mu}^{2}-i 0\right)^{-\frac{1}{2}}\left[\frac{\left(p_{\mu}^{2}-\eta_{1}+\eta_{2}\right)^{2}-4 p_{\mu}^{2} \eta_{2}}{p_{\mu}^{2}}+i 0\right]^{\frac{v-3}{2}}\right. \\
\left.+e^{i \pi(v-2)}\left(p_{\mu}^{2}+i 0\right)^{-\frac{1}{2}}\left[\frac{\left(p_{\mu}^{2}-\eta_{1}+\eta_{2}\right)^{2}-4 p_{\mu}^{2} \eta_{2}}{p_{\mu}^{2}}-i 0\right]^{\frac{v-3}{2}}\right\}
\end{gathered}
$$


With this result we have for (7.3)

$$
\begin{gathered}
h(\rho)=\frac{\pi^{\frac{v-3}{2}}}{2^{v-1}} e^{\frac{i \pi(2-v)}{2}} \Gamma\left(\frac{3-v}{2}\right) \iint_{-\infty}^{\infty} f\left(\rho_{1}\right) g\left(\rho_{2}\right) \times \\
\left\{(\rho-i 0)^{-\frac{1}{2}}\left[\frac{\left(\rho-\rho_{1}-\rho_{2}\right)^{2}-4 \rho_{1} \rho_{2}}{\rho}+i 0\right]^{\frac{v-3}{2}}+e^{i \pi(v-2)} \times\right. \\
\left.(\rho+i 0)^{-\frac{1}{2}}\left[\frac{\left(\rho-\rho_{1}-\rho_{2}\right)^{2}-4 \rho_{1} \rho_{2}}{\rho}-i 0\right]^{\frac{v-3}{2}}\right\} d \rho_{1} \mathrm{~d} \rho_{2}
\end{gathered}
$$

where $\rho=p_{\mu}^{2}$ and $h=f * g$.

When $v=4$ we have

$$
h(\rho)=\frac{\pi}{2 \rho} \int_{-\infty}^{\infty} f\left(\rho_{1}\right) g\left(\rho_{2}\right)\left[\left(\rho-\rho_{1}-\rho_{2}\right)^{2}-4 \rho_{1} \rho_{2}\right]_{+}^{\frac{1}{2}} d \rho_{1} d \rho_{2}
$$

When $v=2 n+1$ we obtain:

$$
\begin{gathered}
h(\rho)=-\frac{i \pi^{n-1}}{2^{2 n}(n-1) !} \iint_{-\infty}^{\infty} f\left(\rho_{1}\right) g\left(\rho_{2}\right)\left[\frac{\left(\rho-\rho_{1}-\rho_{2}\right)^{2}-4 \rho_{1} \rho_{2}}{\rho}\right]^{n-1}\left\{(\rho-i 0)^{-\frac{1}{2}} \times\right. \\
{\left[\psi(n)+\frac{i \pi}{2}+\ln \left[\frac{\left(\rho-\rho_{1}-\rho_{2}\right)^{2}-4 \rho_{1} \rho_{2}}{\rho}+i 0\right]\right]-(\rho+i 0)^{-\frac{1}{2}}} \\
\left.\left[\psi(n)+\frac{i \pi}{2}+\ln \left[-\frac{\left(\rho-\rho_{1}-\rho_{2}\right)^{2}-4 \rho_{1} \rho_{2}}{\rho}+i 0\right]\right]\right\} d \rho_{1} d \rho_{2}
\end{gathered}
$$

As an example we will evaluate the convolution of $\delta\left(\rho-m_{1}^{2}\right)$ with $\delta\left(\rho-m_{2}^{2}\right)$ for $v \neq 2 n+1$. In this case we have:

$$
h(\rho)=\frac{\pi^{\frac{v-3}{2}}}{2^{v-1}} e^{\frac{i \pi(2-v)}{2}}\left\{(\rho-i 0)^{-\frac{1}{2}}\left[\frac{\left(\rho-m_{1}^{2}-m_{2}^{2}\right)^{2}-4 m_{1}^{2} m_{2}^{2}}{\rho}+i 0\right]^{\frac{v-3}{2}}+\right.
$$




$$
\left.e^{i \pi(v-2)}(\rho+i 0)^{-\frac{1}{2}}\left[\frac{\left(\rho-m_{1}^{2}-m_{2}^{2}\right)^{2}-4 m_{1}^{2} m_{2}^{2}}{\rho}-i 0\right]^{\frac{v-3}{2}}\right\}
$$

When $v=4, m_{1}=0, m_{2}=m$ we obtain:

$$
\delta(\rho) * \delta\left(\rho-m^{2}\right)=\frac{\pi}{2 \rho}\left|\rho-m^{2}\right|
$$

If we use the dimension $v$ as a regularizing parameter, we can define the product of two tempered distributions as

$$
\begin{gathered}
\hat{h}(x, v)=(2 \pi)^{v} \hat{f}(x, v) \hat{g}(x, v)=(2 \pi)^{v} \mathcal{F}^{-1}\{f(\rho, v)\} \mathcal{F}^{-1}\{g(\rho, v)\}= \\
\mathcal{F}^{-1}\{f(\rho, v) * g(\rho, v)\}=\mathcal{F}^{-1}\{h(\rho, v)\}
\end{gathered}
$$

where $\mathcal{F}^{-1}$ was defined in section 5 by means of (15.8) and where (7.10) should be re-interpreted as:

$$
\begin{gathered}
h(\rho, v)=\frac{\pi^{\frac{v-3}{2}}}{2^{v-1}} e^{\frac{i \pi(2-v)}{2}} \Gamma\left(\frac{3-v}{2}\right) \iint_{-\infty}^{\infty} f\left(\rho_{1}, v\right) g\left(\rho_{2}, v\right) \times \\
\left\{(\rho-i 0)^{-\frac{1}{2}}\left[\frac{\left(\rho-\rho_{1}-\rho_{2}\right)^{2}-4 \rho_{1} \rho_{2}}{\rho}+i 0\right]^{\frac{v-3}{2}}+e^{i \pi(v-2)} \times\right. \\
\left.(\rho+i 0)^{-\frac{1}{2}}\left[\frac{\left(\rho-\rho_{1}-\rho_{2}\right)^{2}-4 \rho_{1} \rho_{2}}{\rho}-i 0\right]^{\frac{v-3}{2}}\right\} d \rho_{1} d \rho_{2}
\end{gathered}
$$


The same procedure is valid when $\hat{f}(x, v)$ and $\hat{g}(x, v)$ are distributions of exponential type. Here $f(\rho, v)$ and $g(\rho, v)$ are defined by:

$$
\begin{aligned}
& F(\rho, v)=\frac{1}{2 \pi i} \int_{-\infty}^{\infty} \frac{f(t, v)}{t-\rho} d t \\
& G(\rho, v)=\frac{1}{2 \pi i} \int_{-\infty}^{\infty} \frac{g(t, v)}{t-\rho} d t
\end{aligned}
$$

where $\mathrm{F}$ and $\mathrm{G}$ are the tempered ultradistributions given by

$$
\mathrm{F}(\rho, v)=\mathcal{F}\{\hat{\mathrm{f}}(x, v)\} \quad \mathrm{G}(\rho, v)=\mathcal{F}\{\hat{\mathrm{g}}(x, v)\}
$$

This procedure generalize to the Minkowskian space the dimensional regularization in configuration space defined in ref.[13] for the Euclidean space. As an example of the use of this method we give the evaluation of the convolution product of two complex mass Wheeler's propagators. From (5.22) and (5.9) we have:

$$
\begin{gathered}
\mathcal{F}\left\{\mathcal{w}_{\mu_{1}}(x, v) \mathcal{w}_{\mu_{2}}(x, v)\right\}=-\frac{\pi^{2}}{2 \rho} \frac{\left(\mu_{1} \mu_{2}\right)^{\frac{v-2}{2}}}{(2 \pi)^{\frac{v+2}{2}}} \int_{0}^{\infty} x^{\frac{4-v}{2}} \mathcal{J}_{\frac{2-v}{2}}\left(\mu_{1} x\right) \mathcal{J}_{\frac{2-v}{2}}\left(\mu_{2} x\right) \times \\
\left\{\Theta[\Im(\rho)] e^{\frac{i \pi(v-2)}{4}} \mathcal{K}_{\frac{v-2}{2}}\left(-i x \rho^{1 / 2}\right)-\Theta[-\Im(\rho)] e^{\frac{i \pi(2-v)}{4}} \mathcal{K}_{\frac{v-2}{2}}\left(i x \rho^{1 / 2}\right)\right\} d x \quad(7.17
\end{gathered}
$$

To evaluate (7.17) we use

$$
\int_{0}^{\infty} \mathcal{J}_{\frac{2-v}{2}}\left(\mu_{1} x\right) \mathcal{J}_{\frac{2-v}{2}}\left(\mu_{2} x\right) \mathcal{K}_{\frac{v-2}{2}}(x z) d x=
$$




$$
\frac{1}{\sqrt{\pi}} \frac{\Gamma\left(\frac{3-v}{2}\right)}{2^{\frac{3 v-6}{2}}} \frac{z^{\frac{2-v}{2}}}{\left(\mu_{1} \mu_{2}\right)^{\frac{v-2}{2}}}\left[\left(z^{2}+\mu_{1}^{2}+\mu_{2}^{2}\right)^{2}-4 \mu_{1}^{2} \mu_{2}^{2}\right]^{\frac{v-3}{2}}
$$

and to deduce (7.18) we have used:

$$
\mathcal{K}_{\frac{v-2}{2}}(x z)=\frac{1}{2}\left(\frac{z x}{2}\right)^{\frac{v-2}{2}} \int_{0}^{\infty} t^{-\frac{v}{2}} e^{-t-\frac{z^{2} x^{2}}{4 t}} d t
$$

(See 8.432(6) of ref.[9]). Thus from (7.18) we have:

$$
\begin{gathered}
\mathcal{F}\left\{\mathcal{w}_{\mu_{1}}(x, v) \mathcal{w}_{\mu_{2}}(x, v)\right\}=\frac{(2 \pi)^{\frac{1-v}{2}}}{2^{\frac{3 v-1}{2}}} \Gamma\left(\frac{3-v}{2}\right) e^{\frac{i \pi(v-2)}{2}} \times \\
\rho^{\frac{v-2}{2}} \operatorname{Sgn}[\Im(\rho)]\left[\left(\rho-\mu_{1}^{2}-\mu_{2}^{2}\right)^{2}-4 \mu_{1}^{2} \mu_{2}^{2}\right]^{\frac{v-3}{2}}
\end{gathered}
$$

and consequently:

$$
\begin{gathered}
\left\{W_{\mu_{1}}(\rho, v) * W_{\mu_{2}}(\rho, v)\right\}=\frac{(2 \pi)^{\frac{v+1}{2}}}{2^{\frac{3 v-1}{2}}} \Gamma\left(\frac{3-v}{2}\right) e^{\frac{i \pi(v-2)}{2}} \times \\
\rho^{\frac{v-2}{2}} \operatorname{Sgn}[\Im(\rho)]\left[\left(\rho-\mu_{1}^{2}-\mu_{2}^{2}\right)^{2}-4 \mu_{1}^{2} \mu_{2}^{2}\right]^{\frac{v-3}{2}}
\end{gathered}
$$

\subsection{The Convolution of two Lorentz Invariant Tem- pered Ultradistributions}

To obtain a expression for the convolution of two tempered ultradistributions

we consider the formula (7.11). As a first step we extend $h(\rho)$ as tempered 
ultradistribution. For this pourpose we consider the function:

$$
l\left(\rho, \rho_{1}, \rho_{2}\right)=\left[\left(\rho-\rho_{1}-\rho_{2}\right)^{2}-4 \rho_{1} \rho_{2}\right]_{+}^{\frac{1}{2}}
$$

The Fourier antitransform of (17.21) is:

$$
\begin{gathered}
\hat{\imath}\left(x, \rho_{1}, \rho_{2}\right)=\frac{e^{-i\left(\rho_{1}+\rho_{2}\right) x}}{|x|}\left\{\left(\rho_{1} \rho_{2}+i 0\right)^{\frac{1}{2}} \mathcal{N}_{1}\left[2\left(\rho_{1} \rho_{2}+i 0\right)^{\frac{1}{2}}|x|\right]+\right. \\
\left.\Theta\left(-\rho_{1} \rho_{2}\right) \sqrt{-\rho_{1} \rho_{2}} \mathcal{J}_{1}\left(2 i \sqrt{-\rho_{1} \rho_{2}}|x|\right)\right\}
\end{gathered}
$$

where $\mathcal{N}_{1}$ is the Newman function. If we consider now the distribution:

$$
m\left(\rho, \rho_{1}, \rho_{2}\right)=\rho^{-1}\left[\left(\rho-\rho_{1}-\rho_{2}\right)^{2}-4 \rho_{1} \rho_{2}\right]_{+}^{\frac{1}{2}}
$$

the corresponding tempered ultradistribution is:

$$
M\left(\rho, \rho_{1}, \rho_{2}\right)=\frac{1}{2 \pi i} \int_{-\infty}^{\infty} \frac{t^{-1}\left[\left(t-\rho_{1}-\rho_{2}\right)^{2}-4 \rho_{1} \rho_{2}\right]_{+}^{\frac{1}{2}}}{t-\rho} d t
$$

which can also be written as:

$$
\begin{gathered}
M\left(\rho, \rho_{1}, \rho_{2}\right)=\frac{1}{\rho}\left\{\mathcal{F}\{\hat{l}\}\left(\rho, \rho_{1}, \rho_{2}\right)-\right. \\
\left.\frac{1}{2}\left[\mathcal{F}\{\hat{l}\}\left(i 0, \rho_{1}, \rho_{2}\right)+\mathcal{F}\{\hat{l}\}\left(-i 0, \rho_{1}, \rho_{2}\right)\right]\right\}
\end{gathered}
$$

Thus the extension to the complex plane of $h(\rho), N(\rho)$ is:

$$
N(\rho)=\frac{\pi}{2} \iint_{-\infty}^{\infty} f\left(\rho_{1}\right) g\left(\rho_{2}\right) M\left(\rho, \rho_{1}, \rho_{2}\right) d \rho_{1} d \rho_{2}
$$


To obtain $M$ in an explicit way we use the following Laplace transforms:

$$
\begin{gathered}
\mathcal{L}\left\{t^{-1} \mathcal{N}_{1}(a t)\right\}(s)=-\frac{2}{\pi a} \sqrt{s^{2}+a^{2}} \ln \left(\frac{\sqrt{s^{2}+a^{2}}+s}{a}\right)+ \\
\frac{2 s}{a \pi}(\ln (2)+1-\gamma) \\
\mathcal{L}\left\{t^{-1} \mathcal{J}_{1}(a t)\right\}(s)=\frac{\sqrt{s^{2}+a^{2}}-s}{a}
\end{gathered}
$$

(see [14] pags. 310 1nd 313). Then we have for the Fourier transforms:

$$
\begin{gathered}
\mathcal{F}\left\{|t|^{-1} \mathcal{N}_{1}(a|t|)\right\}(\rho)=-\frac{2}{\pi a}\left\{\Theta [ \Im ( \rho ) ] \left[\sqrt{a^{2}-\rho^{2}} \ln \left(\frac{\sqrt{a^{2}-\rho^{2}}-i \rho}{a}\right)+\right.\right. \\
i \rho(\ln (2)+1-\gamma)]-\Theta[-\mathfrak{I}(\rho)]\left[\sqrt{a^{2}-\rho^{2}} \ln \left(\frac{\sqrt{a^{2}-\rho^{2}}+i \rho}{a}\right)-\right. \\
\mathcal{F}\left\{|t|^{-1} \mathcal{J}_{1}(a|t|)\right\}(\rho)=\Theta[\mathfrak{I}(\rho)] \frac{\sqrt{a^{2}-\rho^{2}}-i \rho}{a}- \\
\Theta[-\mathfrak{I}(\rho)] \frac{\sqrt{a^{2}-\rho^{2}}+i \rho}{a}
\end{gathered}
$$

With these results we obtain:

$$
M(\rho)=\Theta[\Im(\rho)]\left\{\Theta\left(\rho_{1} \rho_{2}\right) \sqrt{4 \rho_{1} \rho_{2}-\left(\rho-\rho_{1}-\rho_{2}\right)^{2}} \times\right.
$$




$$
\begin{gathered}
\ln \left[\frac{\sqrt{4 \rho_{1} \rho_{2}-\left(\rho-\rho_{1}-\rho_{2}\right)^{2}}-i\left(\rho-\rho_{1}-\rho_{2}\right)}{2 \sqrt{\rho_{1} \rho_{2}}}\right]+ \\
\Theta\left(-\rho_{1} \rho_{2}\right)\left\{\frac{i \pi}{2}\left[\sqrt{4 \rho_{1} \rho_{2}-\left(\rho-\rho_{1}-\rho_{2}\right)^{2}}-i\left(\rho-\rho_{1}-\rho_{2}\right)\right]+\right. \\
\left.\left.\sqrt{4 \rho_{1} \rho_{2}-\left(\rho-\rho_{1}-\rho_{2}\right)^{2}} \ln \left[\frac{\sqrt{4 \rho_{1} \rho_{2}-\left(\rho-\rho_{1}-\rho_{2}\right)^{2}}-i\left(\rho-\rho_{1}-\rho_{2}\right)}{2 i \sqrt{-\rho_{1} \rho_{2}}}\right]\right\}\right\}- \\
\Theta[-\mathfrak{I}(\rho)]\left\{\Theta\left(\rho_{1} \rho_{2}\right) \sqrt{4 \rho_{1} \rho_{2}-\left(\rho-\rho_{1}-\rho_{2}\right)^{2}} \times\right. \\
\ln \left[\frac{\sqrt{4 \rho_{1} \rho_{2}-\left(\rho-\rho_{1}-\rho_{2}\right)^{2}}+i\left(\rho-\rho_{1}-\rho_{2}\right)}{2 \sqrt{\rho_{1} \rho_{2}}}\right]+ \\
\Theta\left(-\rho_{1} \rho_{2}\right)\left\{\frac{i \pi}{2}\left[\sqrt{4 \rho_{1} \rho_{2}-\left(\rho-\rho_{1}-\rho_{2}\right)^{2}}+i\left(\rho-\rho_{1}-\rho_{2}\right)\right]+\right. \\
\left.\sqrt{4 \rho_{1} \rho_{2}-\left(\rho-\rho_{1}-\rho_{2}\right)^{2}} \ln \left[\frac{\sqrt{4 \rho_{1} \rho_{2}-\left(\rho-\rho_{1}-\rho_{2}\right)^{2}}+i\left(\rho-\rho_{1}-\rho_{2}\right)}{2 i \sqrt{-\rho_{1} \rho_{2}}}\right]\right\} \\
i\left(\rho _ { 1 } \Theta ( - \rho _ { 2 } ) \left[-i \pi\left(\rho_{1}-\rho_{2}\right) \operatorname{Sgn}\left(\rho_{1}+\rho_{2}\right)+2 i \pi \rho_{1} \Theta\left(\rho_{1}+\rho_{2}\right)+\right.\right. \\
\frac{i}{2}\left\{\Theta\left(\rho_{1} \rho_{2}\right)\left(\rho_{1}-\rho_{2}\right) \ln \left(\frac{\rho_{1}}{\rho_{2}}\right)+\Theta\left(-\rho_{1} \rho_{2}\right)\left(\rho_{1}-\rho_{2}\right) \ln \left(-\frac{\rho_{1}}{\rho_{2}}\right)+\right. \\
\Theta\left(-\rho_{1}\right) \Theta\left(\rho_{2}\right)\left[i \pi\left(\rho_{1}-\rho_{2}\right) \operatorname{Sgn}\left(\rho_{1}+\rho_{2}\right)+2 i \pi \rho_{2} \Theta\left(\rho_{1}+\rho_{2}\right)+2 i \pi \rho_{1} \Theta\left(-\rho_{1}-\rho_{2}\right)\right]+
\end{gathered}
$$

To obtain an expression for the convolution of two ultradistribution we use for the Heaviside function the identity:

$$
\Theta(x y)=\Theta(x) \Theta(y)+\Theta(-x) \Theta(-y)
$$


Taking into account that

$$
\Theta(\rho)=\lim _{\Lambda \rightarrow i 0^{+}} \frac{1}{2 \pi i}[\ln (-\rho+\Lambda)-\ln (-\rho-\Lambda)]
$$

a conceptually simple by rather lengthy expression is obtained for Lorentz invariant tempered ultradistributions:

$$
\begin{aligned}
& H_{\lambda}(\rho, \Lambda)=\frac{1}{8 \pi^{2} \rho} \int_{\Gamma_{1}} \int_{\Gamma_{2}} F\left(\rho_{1}\right) G\left(\rho_{2}\right) \rho_{1}^{\lambda} \rho_{2}^{\lambda}\left\{\Theta [ \Im ( \rho ) ] \left\{\left[\ln \left(-\rho_{1}+\Lambda\right)-\ln \left(-\rho_{1}-\Lambda\right)\right] \times\right.\right. \\
& {\left[\ln \left(-\rho_{2}+\Lambda\right)-\ln \left(-\rho_{2}-\Lambda\right)\right] \sqrt{4\left(\rho_{1}+\Lambda\right)\left(\rho_{2}+\Lambda\right)-\left(\rho-\rho_{1}-\rho_{2}-2 \Lambda\right)^{2}} \times} \\
& \ln \left[\frac{\sqrt{4\left(\rho_{1}+\Lambda\right)\left(\rho_{2}+\Lambda\right)-\left(\rho-\rho_{1}-\rho_{2}-2 \Lambda\right)^{2}}-i\left(\rho-\rho_{1}-\rho_{2}-2 \Lambda\right)}{2 \sqrt{\left(\rho_{1}+\Lambda\right)\left(\rho_{2}+\Lambda\right)}}\right]+ \\
& {\left[\ln \left(\rho_{1}+\Lambda\right)-\ln \left(\rho_{1}-\Lambda\right)\right]\left[\ln \left(\rho_{2}+\Lambda\right)-\ln \left(\rho_{2}-\Lambda\right)\right] \times} \\
& \sqrt{4\left(\rho_{1}-\Lambda\right)\left(\rho_{2}-\Lambda\right)-\left(\rho-\rho_{1}-\rho_{2}+2 \Lambda\right)^{2}} \times \\
& \ln \left[\frac{\sqrt{4\left(\rho_{1}-\Lambda\right)\left(\rho_{2}-\Lambda\right)-\left(\rho-\rho_{1}-\rho_{2}+2 \Lambda\right)^{2}}-i\left(\rho-\rho_{1}-\rho_{2}+2 \Lambda\right)}{2 \sqrt{\left(\rho_{1}-\Lambda\right)\left(\rho_{2}-\Lambda\right)}}\right]+ \\
& {\left[\ln \left(\rho_{1}+\Lambda\right)-\ln \left(\rho_{1}-\Lambda\right)\right]\left[\ln \left(-\rho_{2}+\Lambda\right)-\ln \left(-\rho_{2}-\Lambda\right)\right] \times} \\
& \left\{\frac{i \pi}{2}\left[\sqrt{4\left(\rho_{1}+\Lambda\right)\left(\rho_{2}-\Lambda\right)-\left(\rho-\rho_{1}-\rho_{2}\right)^{2}}-i\left(\rho-\rho_{1}-\rho_{2}\right)\right]+\right. \\
& \sqrt{4\left(\rho_{1}+\Lambda\right)\left(\rho_{2}-\Lambda\right)-\left(\rho-\rho_{1}-\rho_{2}\right)^{2}} \times \\
& \left.\ln \left[\frac{\sqrt{4\left(\rho_{1}+\Lambda\right)\left(\rho_{2}-\Lambda\right)-\left(\rho-\rho_{1}-\rho_{2}\right)^{2}}-i\left(\rho-\rho_{1}-\rho_{2}\right)}{2 i \sqrt{-\left(\rho_{1}+\Lambda\right)\left(\rho_{2}-\Lambda\right)}}\right]\right\}+ \\
& {\left[\ln \left(-\rho_{1}+\Lambda\right)-\ln \left(-\rho_{1}-\Lambda\right)\right]\left[\ln \left(\rho_{2}+\Lambda\right)-\ln \left(\rho_{2}-\Lambda\right)\right] \times}
\end{aligned}
$$




$$
\begin{aligned}
& \left\{\frac{i \pi}{2}\left[\sqrt{4\left(\rho_{1}-\Lambda\right)\left(\rho_{2}+\Lambda\right)-\left(\rho-\rho_{1}-\rho_{2}\right)^{2}}-i\left(\rho-\rho_{1}-\rho_{2}\right)\right]+\right. \\
& \sqrt{4\left(\rho_{1}-\Lambda\right)\left(\rho_{2}+\Lambda\right)-\left(\rho-\rho_{1}-\rho_{2}\right)^{2}} \times \\
& \left.\left.\ln \left[\frac{\sqrt{4\left(\rho_{1}-\Lambda\right)\left(\rho_{2}+\Lambda\right)-\left(\rho-\rho_{1}-\rho_{2}\right)^{2}}-i\left(\rho-\rho_{1}-\rho_{2}\right)}{2 i \sqrt{-\left(\rho_{1}-\Lambda\right)\left(\rho_{2}+\Lambda\right)}}\right]\right\}\right\}- \\
& \Theta[-\mathfrak{I}(\rho)]\left\{\left[\ln \left(-\rho_{1}+\Lambda\right)-\ln \left(-\rho_{1}-\Lambda\right)\right]\left[\ln \left(-\rho_{2}+\Lambda\right)-\ln \left(-\rho_{2}-\Lambda\right)\right] \times\right. \\
& \sqrt{4\left(\rho_{1}-\Lambda\right)\left(\rho_{2}-\Lambda\right)-\left(\rho-\rho_{1}-\rho_{2}+2 \Lambda\right)^{2}} \times \\
& \ln \left[\frac{\sqrt{4\left(\rho_{1}-\Lambda\right)\left(\rho_{2}-\Lambda\right)-\left(\rho-\rho_{1}-\rho_{2}+2 \Lambda\right)^{2}}-i\left(\rho-\rho_{1}-\rho_{2}+2 \Lambda\right)}{2 \sqrt{\left(\rho_{1}-\Lambda\right)\left(\rho_{2}-\Lambda\right)}}\right]+ \\
& {\left[\ln \left(\rho_{1}+\Lambda\right)-\ln \left(\rho_{1}-\Lambda\right)\right]\left[\ln \left(\rho_{2}+\Lambda\right)-\ln \left(\rho_{2}-\Lambda\right)\right] \times} \\
& \sqrt{4\left(\rho_{1}+\Lambda\right)\left(\rho_{2}+\Lambda\right)-\left(\rho-\rho_{1}-\rho_{2}-2 \Lambda\right)^{2}} \times \\
& \ln \left[\frac{\sqrt{4\left(\rho_{1}+\Lambda\right)\left(\rho_{2}+\Lambda\right)-\left(\rho-\rho_{1}-\rho_{2}-2 \Lambda\right)^{2}}-i\left(\rho-\rho_{1}-\rho_{2}-2 \Lambda\right)}{2 \sqrt{\left(\rho_{1}+\Lambda\right)\left(\rho_{2}+\Lambda\right)}}\right]+ \\
& {\left[\ln \left(\rho_{1}+\Lambda\right)-\ln \left(\rho_{1}-\Lambda\right)\right]\left[\ln \left(-\rho_{2}+\Lambda\right)-\ln \left(-\rho_{2}-\Lambda\right)\right] \times} \\
& \left\{\frac{i \pi}{2}\left[\sqrt{4\left(\rho_{1}-\Lambda\right)\left(\rho_{2}+\Lambda\right)-\left(\rho-\rho_{1}-\rho_{2}\right)^{2}}-i\left(\rho-\rho_{1}-\rho_{2}\right)\right]+\right. \\
& \sqrt{4\left(\rho_{1}-\Lambda\right)\left(\rho_{2}+\Lambda\right)-\left(\rho-\rho_{1}-\rho_{2}\right)^{2}} \times \\
& \left.\ln \left[\frac{\sqrt{4\left(\rho_{1}-\Lambda\right)\left(\rho_{2}+\Lambda\right)-\left(\rho-\rho_{1}-\rho_{2}\right)^{2}}-i\left(\rho-\rho_{1}-\rho_{2}\right)}{2 i \sqrt{-\left(\rho_{1}-\Lambda\right)\left(\rho_{2}+\Lambda\right)}}\right]\right\}+ \\
& {\left[\ln \left(-\rho_{1}+\Lambda\right)-\ln \left(-\rho_{1}-\Lambda\right)\right]\left[\ln \left(\rho_{2}+\Lambda\right)-\ln \left(\rho_{2}-\Lambda\right)\right] \times} \\
& \left\{\frac{i \pi}{2}\left[\sqrt{4\left(\rho_{1}+\Lambda\right)\left(\rho_{2}-\Lambda\right)-\left(\rho-\rho_{1}-\rho_{2}\right)^{2}}-i\left(\rho-\rho_{1}-\rho_{2}\right)\right]+\right. \\
& \sqrt{4\left(\rho_{1}+\Lambda\right)\left(\rho_{2}-\Lambda\right)-\left(\rho-\rho_{1}-\rho_{2}\right)^{2}} \times
\end{aligned}
$$




$$
\begin{aligned}
& \left.\left.\ln \left[\frac{\sqrt{4\left(\rho_{1}+\Lambda\right)\left(\rho_{2}-\Lambda\right)-\left(\rho-\rho_{1}-\rho_{2}\right)^{2}}-i\left(\rho-\rho_{1}-\rho_{2}\right)}{2 i \sqrt{-\left(\rho_{1}+\Lambda\right)\left(\rho_{2}-\Lambda\right)}}\right]\right\}\right\}-\frac{i}{2} \times \\
& \left\{\left[\ln \left(-\rho_{1}+\Lambda\right)-\ln \left(-\rho_{1}-\Lambda\right)\right]\left[\ln \left(-\rho_{2}+\Lambda\right)-\ln \left(-\rho_{2}-\Lambda\right)\right] \times\right. \\
& \left(\rho_{1}-\rho_{2}\right)\left[\ln \left(i \sqrt{\frac{\rho_{1}+\Lambda}{\rho_{2}+\Lambda}}\right)+\ln \left(-i \sqrt{\frac{\rho_{1}-\Lambda}{\rho_{2}-\Lambda}}\right)\right]+ \\
& {\left[\ln \left(\rho_{1}+\Lambda\right)-\ln \left(\rho_{1}-\Lambda\right)\right]\left[\ln \left(\rho_{2}+\Lambda\right)-\ln \left(\rho_{2}-\Lambda\right)\right] \times} \\
& \left(\rho_{1}-\rho_{2}\right)\left[\ln \left(-i \sqrt{\frac{\Lambda-\rho_{1}}{\Lambda-\rho_{2}}}\right)+\ln \left(i \sqrt{\frac{\Lambda+\rho_{1}}{\Lambda+\rho_{2}}}\right)\right]+ \\
& {\left[\ln \left(\rho_{1}+\Lambda\right)-\ln \left(\rho_{1}-\Lambda\right)\right]\left[\ln \left(-\rho_{2}+\Lambda\right)-\ln \left(-\rho_{2}-\Lambda\right)\right] \times} \\
& \left\{\left(\rho_{1}-\rho_{2}\right)\left[\ln \left(\sqrt{\frac{\Lambda+\rho_{1}}{\Lambda-\rho_{2}}}\right)+\ln \left(\sqrt{\frac{\Lambda-\rho_{1}}{\Lambda+\rho_{2}}}\right)\right]+\right. \\
& \frac{\left(\rho_{1}-\rho_{2}\right)}{2}\left[\ln \left(-\rho_{1}-\rho_{2}+\Lambda\right)-\ln \left(-\rho_{1}-\rho_{2}-\Lambda\right)-\right. \\
& \left.\ln \left(\rho_{1}+\rho_{2}+\Lambda\right)+\ln \left(\rho_{1}+\rho_{2}-\Lambda\right)\right]+\rho_{2}\left[\ln \left(-\rho_{1}-\rho_{2}+\Lambda\right)-\right. \\
& \left.\left.\ln \left(-\rho_{1}-\rho_{2}-\Lambda\right)\right]+\rho_{1}\left[\ln \left(\rho_{1}+\rho_{2}+\Lambda\right)-\ln \left(\rho_{1}+\rho_{2}-\Lambda\right)\right]\right\} \\
& {\left[\ln \left(-\rho_{1}+\Lambda\right)-\ln \left(-\rho_{1}-\Lambda\right)\right]\left[\ln \left(\rho_{2}+\Lambda\right)-\ln \left(\rho_{2}-\Lambda\right)\right] \times} \\
& \left\{\left(\rho_{1}-\rho_{2}\right)\left[\ln \left(\sqrt{\frac{\Lambda-\rho_{1}}{\Lambda+\rho_{2}}}\right)+\ln \left(\sqrt{\frac{\Lambda+\rho_{1}}{\Lambda-\rho_{2}}}\right)\right]+\right. \\
& \frac{\left(\rho_{1}-\rho_{2}\right)}{2}\left[\ln \left(\rho_{1}+\rho_{2}+\Lambda\right)-\ln \left(\rho_{1}+\rho_{2}-\Lambda\right)-\right. \\
& \left.\ln \left(-\rho_{1}-\rho_{2}+\Lambda\right)+\ln \left(-\rho_{1}-\rho_{2}-\Lambda\right)\right]+\rho_{1}\left[\ln \left(-\rho_{1}-\rho_{2}+\Lambda\right)-\right.
\end{aligned}
$$$$
\left.\left.\left.\left.\ln \left(-\rho_{1}-\rho_{2}-\Lambda\right)\right]+\rho_{2}\left[\ln \left(\rho_{1}+\rho_{2}+\Lambda\right)-\ln \left(\rho_{1}+\rho_{2}-\Lambda\right)\right]\right\}\right\}\right\} d \rho_{1} d \rho_{2}
$$ 
Which defines an ultradistribution on the variables $\rho$ and $\Lambda$ for $|\mathfrak{I}(\rho)|>\mathfrak{I}(\Lambda)>\left|\mathfrak{I}\left(\rho_{1}\right)\right|+\left|\mathfrak{I}\left(\rho_{2}\right)\right|$

Let $\mathfrak{i}$ be a vertical band contained in the complex $\lambda$-plane $\mathfrak{A}$. Integral (7.34) is an analytic function of $\lambda$ defined in the domain $\mathfrak{k}$. Moreover, it is bounded by a power of $|\rho \Lambda|$. Then, according to the method of ref. $[8], H_{\lambda}(\rho, \Lambda)$ can be analytically continued to other parts of $\mathfrak{z}$. Thus we define

$$
\begin{gathered}
H(\rho)=H^{(0)}\left(\rho, i 0^{+}\right) \\
H_{\lambda}\left(\rho, i 0^{+}\right)=\sum_{-m}^{\infty} H^{(\mathfrak{n})}\left(\rho, i 0^{+}\right) \lambda^{n}
\end{gathered}
$$

As in the other cases we define now

$$
\{\mathrm{F} * \mathrm{G}\}(\rho)=\mathrm{H}(\rho)
$$

as the convolution of two Lorentz invariant tempered ultradistributions. The proof that $H(\rho)$ is a Tempered Ultradistribution is similar to the one given in ref. [3] for the one-dimensional case. Starting with (7.34) we can write:

$$
H_{\lambda}\left(\rho, i 0^{+}\right)=-\frac{1}{2 \rho} \iint_{-\infty}^{\infty} f_{\lambda}\left(\rho_{1}\right) g_{\lambda}\left(\rho_{2}\right) M\left(\rho . \rho_{1}, \rho_{2}\right) d \rho_{1} d \rho_{2}
$$

where $f_{\lambda}(\rho)$ and $g_{\lambda}(\rho)$ are defined by Dirac's formula:

$$
\rho^{\lambda} F_{\lambda}(\rho)=\frac{1}{2 \pi i} \int_{-\infty}^{\infty} \frac{f_{\lambda}(t)}{t-\rho} d t ; \rho^{\lambda} G_{\lambda}(\rho)=\frac{1}{2 \pi i} \int_{-\infty}^{\infty} \frac{g_{\lambda}(t)}{t-\rho} d t
$$


Let $\widehat{H}_{\lambda}(x)$ be the Fourier antitransform of $\mathrm{H}_{\lambda}\left(\rho, \mathfrak{i} 0^{+}\right)$. The according with (6.12) to (6.17) we can express $\mathrm{H}^{(0)}(\mathrm{x})$ as a function of de Laurent developments of $\hat{f}_{\lambda}(x)$ and $\hat{g}_{\lambda}(x)$

\section{Examples}

As an example of the use of (7.35) we will evaluate the convolution product of $\delta(\rho)$ with $\delta\left(\rho-\mu^{2}\right)$ with $\mu=\mu_{R}+i \mu_{I}$ a complex number such that: $\mu_{R}^{2}>\mu_{I}^{2}$, $\mu_{R} \mu_{I}>0$. Thus from (7.34) we obtain:

$$
\begin{gathered}
\mathrm{H}_{0}(\rho, \Lambda)=-i \pi\left[\ln \left(-\mu^{2}+\Lambda\right)-\ln \left(-\mu^{2}+\lambda\right)\right]\left\{\frac { i ( \rho - \mu ^ { 2 } ) } { 8 \pi ^ { 2 } \rho } \left[\ln \left(\frac{\rho-\mu^{2}}{\sqrt{\Lambda\left(\mu^{2}+\Lambda\right)}}\right)+\right.\right. \\
\left.\left.\ln \left(\frac{\mu^{2}-\rho}{\sqrt{-\Lambda\left(\mu^{2}+\Lambda\right)}}\right)\right]+\frac{\mu^{2}-\rho}{16 \pi \rho}\right\}-i \pi\left[\ln \left(-\mu^{2}+\Lambda\right)-\ln \left(-\mu^{2}+\lambda\right)\right] \times \\
\left\{\frac{\left.-i \mu^{2}\right)}{8 \pi^{2} \rho}\left[\ln \left(\sqrt{\frac{\Lambda}{\mu^{2}+\Lambda}}\right)+\ln \left(\sqrt{\frac{\Lambda}{\Lambda-\mu^{2}}}\right)\right]-\frac{\mu^{2}}{16 \pi \rho}\right\}
\end{gathered}
$$

Simplifying terms (7.47) turns into:

$$
\begin{aligned}
H_{0}(\rho, \Lambda)= & -i \pi\left[\ln \left(-\mu^{2}+\Lambda\right)-\ln \left(-\mu^{2}+\lambda\right)\right]\left\{\frac { i ( \rho - \mu ^ { 2 } ) } { 8 \pi ^ { 2 } \rho } \left[\ln \left(\rho-\mu^{2}\right)+\right.\right. \\
& \left.\left.\ln \left(\mu^{2}-\rho\right)\right]+\frac{i \mu^{2}}{8 \pi^{2} \rho}\left[\ln \left(\mu^{2}+\Lambda\right)+\ln \left(\mu^{2}-\Lambda\right)\right]\right\}
\end{aligned}
$$

Now, if

$$
F_{1}(\mu, \Lambda)=\ln \left(-\mu^{2}+\Lambda\right)-\ln \left(-\mu^{2}-\Lambda\right)
$$


then

$$
\mathrm{F}_{1}\left(\mu, \mathrm{i} 0^{+}\right)=2 \mathrm{i} \pi ; \mu_{\mathrm{R}}^{2}>\mu_{\mathrm{I}}^{2} ; \mu_{\mathrm{R}} \mu_{\mathrm{I}}>0
$$

And, if

$$
F_{2}(\mu, \Lambda)=\ln \left(\mu^{2}+\Lambda\right)-\ln \left(\mu^{2}-\Lambda\right)
$$

then

$$
\mathrm{F}_{2}\left(\mu, i 0^{+}\right)=0 ; \mu_{\mathrm{R}}^{2}>\mu_{\mathrm{I}}^{2} ; \mu_{\mathrm{R}} \mu_{\mathrm{I}}>0
$$

Using these results we obtain:

$$
H(\rho)=\frac{i\left(\rho-\mu^{2}\right)}{4 \rho}\left[\ln \left(\rho-\mu^{2}\right)+\ln \left(\mu^{2}-\rho\right)\right]+\frac{i \mu^{2}}{2 \rho} \ln \left(\mu^{2}\right)
$$

As an example of the use of (6.17) we will evaluate the convolution product of two Dirac's delta: $\delta(\rho) * \delta(\rho)$. In this case we have:

$$
F_{\lambda}(\rho)=-\frac{\rho^{\lambda-1}}{2 \pi i}
$$

and as a consequence:

$$
f_{\lambda}(\rho)=\frac{\sin (\pi \lambda)}{\pi} \rho_{-}^{\lambda-1}
$$

The Fourier antitransform of (7.44) is:

$$
\hat{f}_{\lambda}(x)-\frac{2^{2 \lambda}}{4 \pi^{3}} \frac{\Gamma(1+\lambda)}{\Gamma(1-\lambda)}\left[x_{+}^{-\lambda-1}-\cos (\pi \lambda) x_{-}^{-\lambda-1}\right]
$$

which can be written as:

$$
\hat{f}_{\lambda}(x)-\frac{2^{2 \lambda}}{4 \pi^{3}} \frac{\Gamma(1+\lambda)}{\Gamma(1-\lambda)}\left[\frac{\cos (\pi \lambda)-1}{\lambda} \delta(x)+x_{+}^{-1}-\cos (\pi \lambda) x_{-}^{-1}+\right.
$$




$$
\left.S_{+}^{-\lambda-1}-\cos (\pi \lambda) S_{-}^{-\lambda-1}\right]
$$

Thus we have:

$$
\begin{aligned}
& \hat{f}_{\lambda}^{2}(x)-\frac{2^{4 \lambda}}{16 \pi^{6}} \frac{\Gamma^{2}(1+\lambda)}{\Gamma^{2}(1-\lambda)}\left\{\frac{(\cos (\pi \lambda)-1)^{2}}{\lambda^{2}} \delta^{2}(x)+x_{+}^{-2}+\cos ^{2}(\pi \lambda) x_{-}^{-2}+\right. \\
& {\left[S_{+}^{-\lambda-1}-\cos (\pi \lambda) S_{-}^{-\lambda-1}\right]^{2}+2\left[x_{+}^{-1}-\cos (\pi \lambda) x_{-}^{-1}\right]\left[S_{+}^{-\lambda-1}-\cos (\pi \lambda) S_{-}^{-\lambda-1}\right]+} \\
& \left.2\left[\frac{\cos (\pi \lambda)-1}{\lambda} \delta(x)\right]\left[x_{+}^{-1}-\cos (\pi \lambda) x_{-}^{-1}+S_{+}^{-\lambda-1}-\cos (\pi \lambda) S_{-}^{-\lambda-1}\right]\right\}
\end{aligned}
$$

From (7.47) we obtain:

$$
\lim _{\lambda \rightarrow 0} \hat{f}_{\lambda}^{2}(x)=\frac{4}{(2 \pi)^{6}} x^{-2}
$$

and taking into account that:

$$
\mathcal{F}\left\{x^{-2}\right\}=\frac{\pi^{3}}{2} \operatorname{Sgn}(\rho)
$$

we obtain

$$
\delta(\rho) * \delta(\rho)=\frac{\pi}{2} \operatorname{Sgn}(\rho)
$$




\section{Discussion}

In a earlier paper [3] we have shown the existence of the convolution of two one-dimensional tempered ultradistributions. In other paper ref. [4] we have extended these procedure to n-dimensional space. In four-dimensional space we have given an expression for the convolution of two tempered ultradistributions even in the variables $k^{0}$ and $\rho$. In this paper we obtain a expression for the convolution of two Lorentz invariant tempered ultradistributions in both, Euclidean and Minkowskian space. In an intermediate step of deduction we obtain the generalization to the Minkowskian space of the dimensional regularization in configuration space (ref. [13])

When we use the perturbative development in Quantum Field Theory, we have to deal with products of distributions in configuration space, or else, with convolutions in the Fourier transformed p-space. Unfortunately, products or convolutions ( of distributions ) are in general ill-defined quantities. However, in physical applications one introduces some "regularization" scheme, which allows us to give sense to divergent integrals. Among these procedures we would like to mention the dimensional regularization method ( ref. [15, 16] ). Essentially, the method consists in the separation of the volume element $\left(d^{v} p\right)$ into an angular factor $(d \Omega)$ and a radial factor ( 
$\left.p^{v-1} d p\right)$. First the angular integration is carried out and then the number of dimensions $v$ is taken as a free parameter. It can be adjusted to give a convergent integral, which is an analytic function of $v$.

Our formula (7.34) is similar to the expression one obtains with dimensional regularization. However, the parameter $\lambda$ is completely independents of any dimensional interpretation.

All ultradistributions provide integrands ( in (7.34) ) that are analytic functions along the integration path. The parameter $\lambda$ permits us to control the possible tempered asymptotic behavior ( cf. eq. (3.9) ). The existence of a region of analyticity in $\lambda$, and a subsequent continuation to the point of interest ( ref. 3] ), defines the convolution product.

The properties described below show that tempered ultradistributions provide an appropriate framework for applications to physics. Furthermore, they can "absorb" arbitrary pseudo-polynomials, thanks to eq. (3.10). A property that is interesting for renormalization theory. For this reason and also for the benefit of the reader we began this paper with a summary of the main characteristics of n-dimensional tempered ultradistributions and their Fourier transformed distributions of the exponential type.

As a final remark we would like to point out that our formula for convo- 
lutions is a definition and not a regularization method. 


\section{References}

[1] J. Sebastiao e Silva : Math. Ann. 136, 38 (1958).

[2] M. Hasumi: Tôhoku Math. J. 13, 94 (1961).

[3] C. G. Bollini, T. Escobar and M. C. Rocca : Int. J. of Theor. Phys. 38, 2315 (1999).

[4] C. G Bollini and M.C. Rocca : "Convolution of n-dimensional tempered ultradistributions and Field Theory": hep-th /0309271. To be published in Int. J. of Theor. Phys.

[5] I. M. Gel'fand and N. Ya. Vilenkin : "Generalized Functions" Vol. 4. Academic Press (1964).

[6] I. M. Gel'fand and G. E. Shilov : "Generalized Functions" Vol. 2. Academic Press (1968).

[7] L. Schwartz : "Théorie des distributions". Hermann, Paris (1966).

[8] I. M. Gel'fand and G. E. Shilov : "Generalized Functions" Vol. 1 Ch.1, section 3. Academic Press (1964). 
[9] L. S. Gradshtein and I. M. Ryzhik : "Table of Integrals, Series, and Products". Sixth edition. Academic Press (2000).

[10] G. N. Watson : "A Treatise on the Theory of Bessel Functions" Second edition. Cambridge University Press (1995)

[11] I. M. Gel'fand and G. E. Shilov : "Generalized Functions" Vol. 1. Academic Press (1964).

[12] A. Erdelyi : "Bateman Manuscript Project" Vol 1 : Higher Transcendental Functions. McGraw-Hill Book Company, Inc. (1953).

[13] C. G. Bollini and J.J Giambiagi : Phys. Rev. D 53, 5761 (1996).

[14] Y. A. Brychkov and A. P. Prudnikov : "Integral Transforms of Generalized Functions". Gordon and Breach Science Publishers. (1989).

[15] C. G. Bollini and J. J. Giambiagi: Phys. Lett. B 40, 566 (1972). Il Nuovo Cim. B 12, 20 (1972).

[16] G.'t Hooft and M. Veltman: Nucl. Phys. B 44, 189 (1972). 